\title{
Implications of path tolerance and path characteristics on critical vehicle manoeuvres
}

Kristoffer Lundahl, Erik Frisk and Lars Nielsen

The self-archived postprint version of this journal article is available at Linköping University Institutional Repository (DiVA):

http:/ / urn.kb.se/ resolve?urn=urn:nbn:se:liu:diva-142995

N.B.: When citing this work, cite the original publication.

This is an electronic version of an article published in:

Lundahl, K., Frisk, E., Nielsen, L., (2017), Implications of path tolerance and path characteristics on critical vehicle manoeuvres, Vehicle System Dynamics, 55(12), 1909-1945.

https:// doi.org/ 10.1080/ 00423114.2017.1339891

Original publication available at:

https:/ / doi.org/ 10.1080/00423114.2017.1339891

Copyright: Taylor \& Francis (STM, Behavioural Science and Public Health Titles)

http:// www.tandf.co.uk/journals/ default.asp 
To appear in Vehicle System Dynamics

Vol. 00, No. 00, Month 20XX, 1-36

\title{
Implications of Path Tolerance and Path Characteristics on Critical Vehicle Maneuvers
}

\author{
K. Lundahl*, E. Frisk, and L. Nielsen \\ Division of Vehicular Systems, Department of Electrical Engineering, Linköping University, \\ Linköping, Sweden \\ ( $v 1.0$ released March 2016$)$
}

\begin{abstract}
Path planning and path following are core components in safe autonomous driving. Typically a path planner provides a path with some tolerance on how tightly the path should be followed. Based on that, and other path characteristics, for example sharpness of curves, a speed profile needs to be assigned so that the vehicle can stay within the given tolerance without going unnecessarily slow. Here, such trajectory planning is based on optimal control formulations where critical cases arise as on-the-limit solutions. The study focuses on heavy commercial vehicles, causing rollover to be of a major concern, due to the relatively high center of gravity. Several results are obtained on required model complexity depending on path characteristics, for example quantification of required path tolerance for a simple model to be sufficient, quantification of when yaw inertia needs to be considered in more detail, and how the curvature rate of change interplays with available friction. Overall, in situations where the vehicle is subject to a wide range of driving conditions, from good transport roads to more tricky avoidance maneuvers, the requirements on the path following will vary. For this, the provided results form a basis for real-time path following.
\end{abstract}

Keywords: Clothiod curve; double lane change; rollover; optimal control; vehicle dynamics; path following;

\section{Introduction}

Path following, or equivalently path tracking, is a fundamental function in most motion control, and it therefore shows up in robotics [6, 20], mobile robots [2, 21], and vehicles $[13,16]$. The typical situation in large complex or autonomous motion control is that the system is split into low- and high-level subsystems, structured in a hierarchical framework $[12,24]$, so that the subsystems are designed to handle specific tasks, such as path planning, path following, or control allocation, see Figure 1. For all these subsystems, and specifically for trajectory planning for path following studied here, they need to run in real-time which means that the design needs to consider computational feasibility which in turn means that one has to consider the balance between model complexity and performance requirements, without sacrificing accuracy. Examples of successful demonstrations of fully-autonomous vehicle systems, with the ability to handle a variety of challenging driving situations, has already been developed and showcased, for example in the Grand Challenge and the Urban Challenge conducted by DARPA [7, 8], Google's self-driving cars [13], Stanford's self-driving Audi [16], to name just a few.

\footnotetext{
*Corresponding author. Email: lundahl.kristoffer@gmail.com
} 


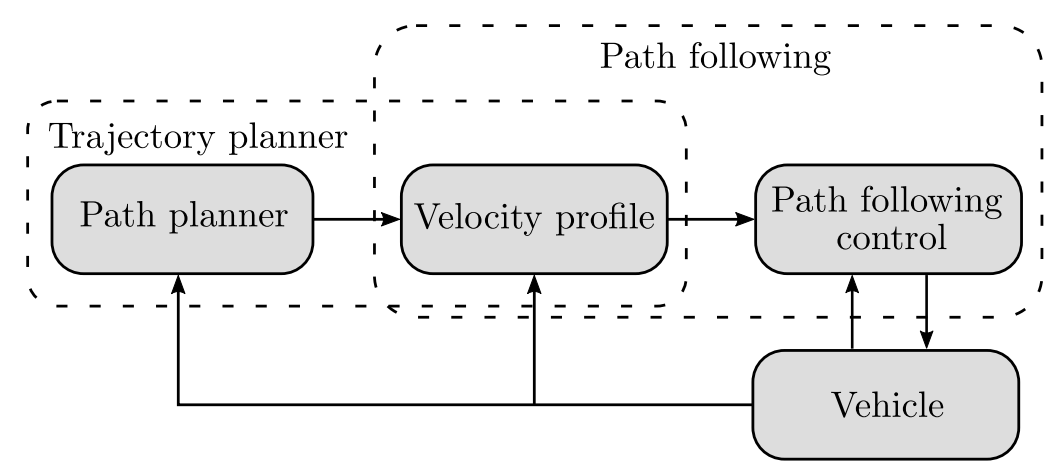

Figure 1. Sketch of overall system, specifically illustrating the relation between path planning and trajectory planning.

Focusing on path following, strictly speaking, it is a restriction of the motion to follow a one-dimensional curve. In practice, both in applications for autonomous vehicles and safety maneuvers, the requirement is that the path is to be followed within some tolerance. In human driving it is obvious that lane keeping on regular roads, where the mass center of the vehicle may vary with tolerances of say $80 \mathrm{~cm}$, is rather easy even at high speed. Precision driving with a maximum tolerance for path deviation of $1 \mathrm{~cm}$ is difficult, and high speed driving with such precision is very difficult for any human.

The present paper puts forward the question of how this type of reasoning transfers to autonomous driving or autonomous critical maneuvers, and importantly what requirements it puts on the system in terms of modeling and control. Intuitively autonomous maneuvers at high speed with a tolerance of $1 \mathrm{~cm}$ require precise modeling and control, and this means that it is natural to study the influence of path tolerance. However, also other path characteristics are important, and it is reasonable to consider how demanding a path is, for example how sharp a curve is or how abrupt an evasive maneuver is. The overall goal of the research program presented in this paper is to give quantitative results that can serve as basis when designing path following systems.

\section{Applications and results}

The problem formulation in this paper is of large general interest, but also quite wide in its full extent regarding all aspects. The choices for models studied, path tolerances studied, and other parameter choices in this paper is to a large extent motivated by the iQMatic project that was initiated in 2013 as a joint collaboration between Scania, Saab, Autoliv, Linköping University and the Royal Institute of Technology. The aim is to develop a fully autonomous heavy vehicle aimed for confined areas, specifically mining applications. Although commercial heavy trucks motivate this study, the vehicle modeling is general and suitable for other types of vehicles. Thus, the method is of interest for a wide variety of vehicles, and the obtained results relevant for vehicle configurations with proportionally similar mass and inertia distributions. The paths obtained from the path planner needs to be given speed profiles, with respect to the tolerance of the path and other path characteristics. Since driving conditions differ from good transport roads on the site to trickier maneuvering in loading cycles around other machines and vehicles, and also workers on the site, the tolerances and characteristics requirements differ over a wide range. Further, since productivity is essential there is an underlying need for real-time and reasonably high speed. Therefore, tables etcetera that give guidance on required model complexity depending on path characteristics is of significant use. Such tables and results are presented in this paper. 


\subsection{Outline of paper}

Regarding maneuvers to study, both a regular path and an evasive maneuver have been selected. The regular path is a clothoid curve, for which the influence of the minimum curvature and the curvature rate of change are studied, for path deviation tolerances ranging from 1 to $80 \mathrm{~cm}$. The evasive maneuver is a double lane change where the size of the maneuver is varied. The geometry of the studied maneuvers is detailed in Section 2.

In path following with large tolerances and smooth path curvatures, for example a simple case of lane keeping, it suffices with simple models. In more critical situations for regular passenger cars, maneuvering is mainly a matter of balancing on the limit set by the available friction. For commercial trucks, and other vehicles having a high center of gravity compared to wheel-track width, rollover is a concern under these circumstances [11, 19]. These aspects are handled by the models introduced in Sections 3 and 4.

Optimal control methods have emerged as a powerful tool since the performance potential of modeling concepts, configurations, and strategies can be evaluated with relative ease in a systematic manner $[4,25]$. Further, these methods have the last few years seen a dramatic increase in performance both regarding computation time and tractable size of feasible problems. Specifically, questions around critical maneuvers can be studied by the optimal solutions to formulations of minimum time or maximum velocity since the solutions obtained typically are on-the-limit. Here, the questions are evaluated by formalizing and solving the problem based on a methodology similar to [4]. The detailed formulation is found in Section 5. The results, their interpretation, discussion, and conclusions are found in Sections 6, 7, and 8.

\section{Maneuvers}

Two different driving maneuvers representing important scenarios that an automated driving system may be subjected to are evaluated in this study. The first one, here called the clothoid turn, is intended to capture the nature of ordinary road turns, which often are designed with clothoid shapes [17]. The second maneuver, the double lane change, simulates a scenario where an object suddenly appears along the driving path, forcing the vehicle into an avoidance maneuver. A range of differently scaled variants of these two maneuvers have been used to investigate the various phenomena they trigger and if conclusions about the vehicle model accuracy can be drawn based on the road or path characteristics. The purpose is to cover two main aspects of autonomous driving, e.g. in a mine setting, where there are plans during normal operation where clothoids are a good representative of plans generated. There is also a need for evasive maneuvers, and there the double lane change is a good representative. In both cases it is interesting to study the effect of motion tolerances and aggressiveness, which in an application would be given by the situation that has occurred. In both cases it is useful to have an understanding and some quantification of the motion control task at hand.

\subsection{Clothoid turn}

Clothoids, or Euler spirals, are curves with a linearly varying curvature, that when used for vehicle path or road design gives a smooth transition from straight line driving. The clothoid shaped path used here is shown in Figure 2, and is divided into three segments; section 1 which is a straight between $s_{0}$ and $s_{1}$, section 2 between $s_{1}$ and $s_{2}$ 
with a linearly increasing curvature, and finally section 3 having a linearly decreasing curvature. The maximum path curvature is found at the $s_{2}$ mark, corresponding to the minimum curve radius $R_{\min }$. The path curvature, denoted $C$, is a function of the curvilinear abscissa $s[10,18]$ (that is, the distance along the path), see Figure 2, and specific for each section according to

$$
\begin{aligned}
& C_{1}(s)=0, \\
& C_{2}(s)=\frac{1}{R_{\min } \Delta s}\left(s-s_{1}\right), \\
& C_{3}(s)=\frac{1}{R_{\min }}-\frac{1}{R_{\min } \Delta s}\left(s-s_{2}\right),
\end{aligned}
$$

where $C_{1}-C_{3}$ refers to the curvature of section $1-3$, and $\Delta s$ is equal to the path distance between $s_{1}$ and $s_{2}$. A continuous expression for the curvature $C(s)$, over the full range of $s$, can then be formulated as

$$
C(s)=\sum_{k=1}^{3} h_{k}(s) C_{k}(s),
$$

where $h_{k}$ is a smooth switching function, being $h_{k}=1$ in section $k$ and otherwise $h_{k}=0$, described using sigmoid functions according to

$$
h_{k}(s)=\frac{1}{1+e^{-\left(s-s_{k}\right)}}-\frac{1}{1+e^{-\left(s-s_{k+1}\right)}} .
$$

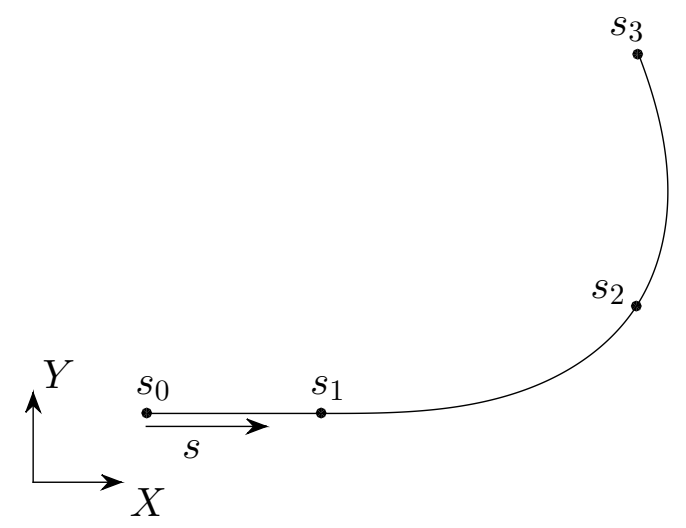

Figure 2. The shape of the clothoid turn is here illustrated, with the marks $s_{0}-s_{3}$ dividing the path into three sections, each with a separate curvature function. The curvilinear abscissa $s$ describes the position along the path. 
The vehicle position, relative the clothoid path, can then be expressed by the following dynamics

$$
\begin{aligned}
& \dot{s}=\frac{v \cos \left(\psi-\psi_{s}\right)}{1-e C(s)}, \\
& \dot{\psi}_{s}=\dot{s} C(s) \\
& \dot{e}=v \sin \left(\psi-\psi_{s}\right) .
\end{aligned}
$$

Thus, $s$ describes the vehicle's position along the path, $e$ the vehicle's perpendicular deviation from the path, $\psi_{s}$ is the tangential direction of the path, $\psi$ the vehicle's yaw angle, and $v$ the absolute vehicle velocity. Representing the path geometry and vehicle position with this formulation enables the user to define a maximum path deviation, or path deviation tolerance, $e_{\max }$ by introducing the constraint

$$
|e| \leq e_{\max }
$$

\subsubsection{Geometric variations of the maneuver}

Since different path characteristics can lead to different model requirements, various geometric variations of the clothoid turn is considered. This is carried out by varying the path parameters; minimum path radius $R_{\text {min }}$, maximum path curvature rate $\left(\frac{\mathrm{d} C}{\mathrm{~d} s}\right)_{\text {max }}$, and path deviation tolerance $e_{\max }$, according to:

- The minimum path radius, $R_{\min } \in\{15,20,25,30,40,50\} \mathrm{m}$.

- The maximum allowed path deviation, $e_{\max } \in\{1,5,10,20,40,80\} \mathrm{cm}$.

- The maximum path curvature rate, $\left(\frac{\mathrm{d} C}{\mathrm{~d} s}\right)_{\max } \in\{0.3,0.5,1,1.5,2,2.5,3\} \cdot 10^{-3} \mathrm{~m}^{-2}$.

The first two variations are performed by simply assigning $R_{\min }$ and $e_{\max }$ the intended values. The curvature rate is adjusted by changing the value of $\Delta s$ in (2) and (3), since the maximum curvature rate is given by

$$
\frac{\mathrm{d} C}{\mathrm{~d} s} \leq \frac{1}{R_{\min } \Delta s}=\left(\frac{\mathrm{d} C}{\mathrm{~d} s}\right)_{\max } .
$$

The geometric effects the minimum radius $R_{\min }$ and maximum path-curvature rate $\left(\frac{\mathrm{d} C}{\mathrm{~d} s}\right)_{\max }$ have on the path are shown in Figures 3(a) and 3(b). Note that changing the curvature rate also affect the length of the path, where for example a slow rate requires a longer distance to reach the minimum radius.

\subsection{Double lane change}

Variations of the double lane-change maneuver is often used as a performance indication of a vehicle's lateral dynamics, as it emulates sudden avoidance maneuvers that can occur in real traffic situations. See, for example, the standardized test ISO 3888-2 [15] which is designed for this purpose. One way of studying this maneuver, for different tolerances and degrees of aggressiveness, would be to define the path as the middle of ISO 3888-2, and then vary the tolerance and geometric path parameters. This would have meant using different versions of polygons implying some complexity of the formulation and also some non-smoothness of the solution space. An effective alternative is instead to define the forbidden domain for the vehicle as depicted in Figure 4. The black rectangle 
Table 1. The dimensions, seen in Figure 4, for the different layout variations of the double lane-change maneuver.

\begin{tabular}{cccc}
\hline $\begin{array}{c}\text { Obstacle width, } \\
w_{o b}[\mathrm{~m}]\end{array}$ & $\begin{array}{c}\text { Obstacle length, } \\
l_{o b}[\mathrm{~m}]\end{array}$ & $\begin{array}{c}\text { Track width, } \\
W_{D L C}[\mathrm{~m}]\end{array}$ & $\begin{array}{c}\text { Track length, } \\
L_{D L C}[\mathrm{~m}]\end{array}$ \\
\hline 2 & 8 & 2.5 & 80 \\
4 & 8 & 5 & 80 \\
6 & 8 & 7.5 & 80 \\
8 & 8 & 10 & 80 \\
\hline
\end{tabular}

represents the outer boundary within the vehicle is limited to operate, and the gray areas represent the objects to avoid.

\subsubsection{Variations of the maneuver}

To evaluate different maneuver characteristics for the double lane change, the maneuver properties are varied in the following two ways:

- The width of the obstacles, $w_{o b} \in\{2,4,6,8\} \mathrm{m}$.

- The friction coefficient of the road surface, $\lambda_{\mu} \in\{0.4,0.6,0.8,1.0,1.2\}$.

When varying the width of the obstacles, all the other dimensions in the $Y$ direction are proportionally scaled, that is, the ratio $\frac{w_{o b}}{W_{D L C}}$ is maintained. The dimensions for all geometry variations of the double lane-change maneuver are listed in Table 1.

The friction coefficient is different in the longitudinal and lateral directions, but is here scaled proportionally with the scaling parameter $\lambda_{\mu}$, according to

$$
\begin{aligned}
& \mu_{x}=\lambda_{\mu} \mu_{x}^{*}, \\
& \mu_{y}=\lambda_{\mu} \mu_{y}^{*},
\end{aligned}
$$

where $\mu$ symbolizes the scaled friction coefficients used in the vehicle models and $\mu^{*}$ the original coefficients that can be found in Table 3 .

\section{Vehicle models}

The vehicle motions and characteristics are represented by three separate vehicle models, in descending order of complexity referred to as; the double track model, the planar no-

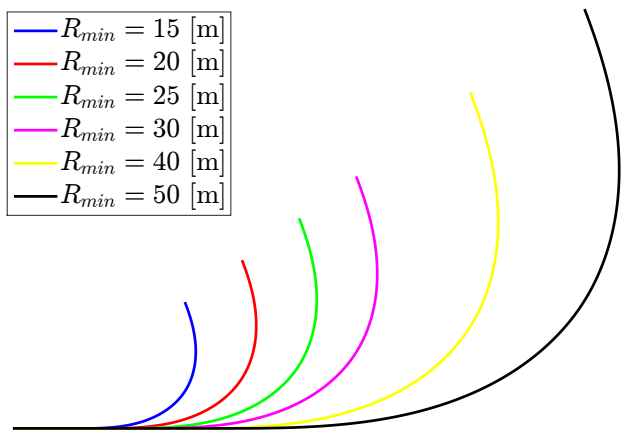

(a) Paths with various minimum radii $R_{m i n}$.

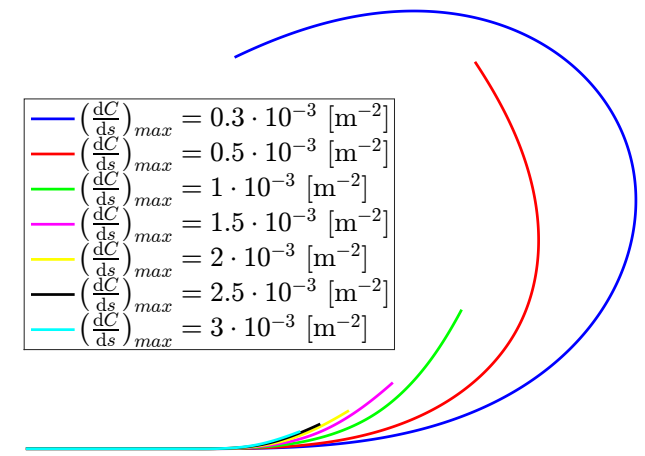

(b) Paths with various curvature rates $\left(\frac{\mathrm{d} C}{\mathrm{~d} s}\right)_{\max }$.

Figure 3. The geometry of the clothoid turn's path for different minimum radii (a) and path curvature rates (b). 


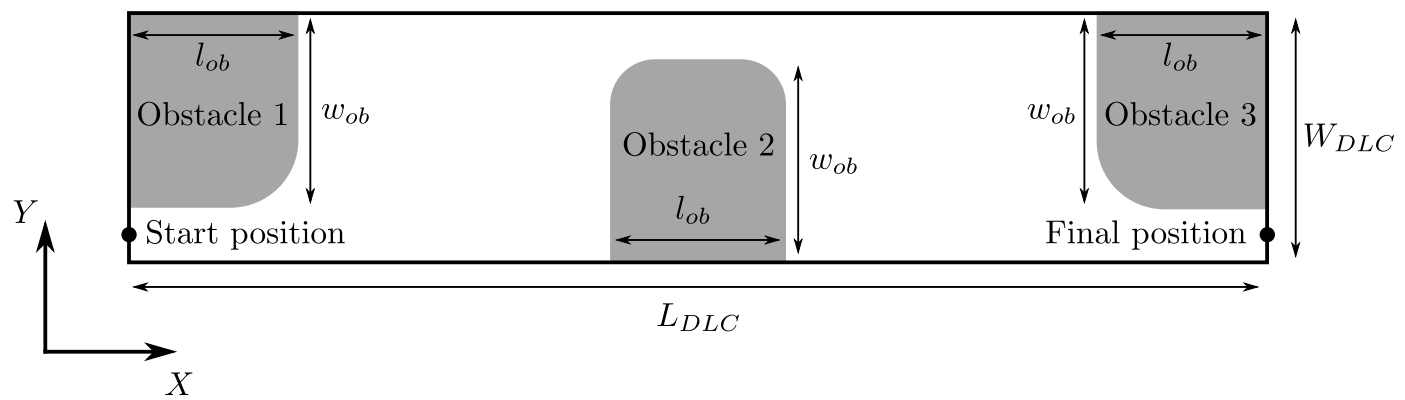

Figure 4. Sketch of the double lane-change maneuver layout. By defining forbidden areas it provides an effective parametrization of a set of maneuvers similar to the standardized double lane change ISO 3888-2. An example can be seen in Figure 19.

slip model, and the static model. The double track model is, in this context, a fairly complex model that captures many of the vehicle specific characteristics. It is therefore here seen as a reference model, which the less complex models are evaluated towards in terms of maximum velocity profiles and driving paths in critical driving scenarios. Note that since the double track model and the planar no-slip model are to be employed in the dynamic optimal-control problems, described in detail in Section 5, and therefore need to be smooth and twice continuously differentiable. The following of this section describes these models in detail.

\subsection{Double track model}

The double track model assumes four individual wheels (two tracks), as illustrated in Figure 5, where the two front wheels are controlled by the steer angle $\delta$. The vehicle body is represented by a single mass element, having the total mass $m$ and the inertia $\mathbf{I}=\operatorname{diag}\left(I_{x x}, I_{y y}, I_{z z}\right)$. The vehicle suspension is represented by suspending the body as a double pendulum. The body can rotate in the roll direction $\phi$ around the roll center, denoted rc in Figure 5, and in the pitch direction $\theta$ around the pitch center, denoted pc. By this arrangement, the roll and pitch centers, which originally are determined from the suspension linkage geometries, can be placed independently of each other. Rotational spring and damper elements are placed in the roll center, with stiffness $K_{\phi}$ and damping coefficient $D_{\phi}$, and pitch center, with the coefficients $K_{\theta}$ and $D_{\theta}$. The pitch center is here located in the ground plane, with the roll center right above at a height of $h_{r c}$, and the chassis body center-of-gravity at the height $h_{c g}$. The body's position $\mathbf{r}_{b}$ can thus be expressed as

$$
\mathbf{r}_{b}=R_{\theta}\left(R_{\phi}\left[\begin{array}{c}
0 \\
0 \\
h_{c g}-h_{r c}
\end{array}\right]+\left[\begin{array}{c}
0 \\
0 \\
h_{r c}
\end{array}\right]\right)
$$

where $R_{\phi}$ and $R_{\theta}$ are roll and pitch rotation matrices according to

$$
R_{\phi}=\left[\begin{array}{ccc}
1 & 0 & 0 \\
0 & \cos \phi & -\sin \phi \\
0 & \sin \phi & \cos \phi
\end{array}\right], \quad R_{\theta}=\left[\begin{array}{ccc}
\cos \theta & 0 & \sin \theta \\
0 & 1 & 0 \\
-\sin \theta & 0 & \cos \theta
\end{array}\right]
$$

The motion dynamics is derived with Lagrange's equations, using the generalized motion variables longitudinal velocity $v_{x}$, lateral velocity $v_{y}$, roll angle $\phi$, pitch angle $\theta$, and 
yaw angle $\psi$, by solving the following system of differential equations

$$
\begin{aligned}
& \frac{\mathrm{d}}{\mathrm{dt}} \frac{\partial \mathcal{L}}{\partial v_{x}}-\dot{\psi} \frac{\partial \mathcal{L}}{\partial v_{y}}=F_{x}, \\
& \frac{\mathrm{d}}{\mathrm{dt}} \frac{\partial \mathcal{L}}{\partial v_{y}}+\dot{\psi} \frac{\partial \mathcal{L}}{\partial v_{x}}=F_{y}, \\
& \frac{\mathrm{d}}{\mathrm{dt}} \frac{\partial \mathcal{L}}{\partial \dot{\phi}}-\frac{\partial \mathcal{L}}{\partial \phi}=M_{\phi}, \\
& \frac{\mathrm{d}}{\mathrm{dt}} \frac{\partial \mathcal{L}}{\partial \dot{\theta}}-\frac{\partial \mathcal{L}}{\partial \theta}=M_{\theta}, \\
& \frac{\mathrm{d}}{\mathrm{dt}} \frac{\partial \mathcal{L}}{\partial \dot{\psi}}-\frac{\partial \mathcal{L}}{\partial \psi}=M_{\psi},
\end{aligned}
$$

where $\mathcal{L}$ is the Lagrangian, composed by the kinetic energy $\mathcal{T}$ and the potential energy $\mathcal{V}$, according to

$$
\begin{aligned}
& \mathcal{L}=\mathcal{T}-\mathcal{V}, \\
& \mathcal{T}=\frac{1}{2} m \mathbf{v}_{b}^{T} \mathbf{v}_{b}+\frac{1}{2} \boldsymbol{\omega}_{b}^{T} \mathbf{I} \boldsymbol{\omega}_{b}, \\
& \mathcal{V}=m g\left(h_{r c} \cos \theta+\left(h_{c g}-h_{r c}\right) \cos \phi\right) .
\end{aligned}
$$

Here, $\mathbf{v}_{b}$ and $\boldsymbol{\omega}_{b}$ are the translational and rotational velocities of the body, and $g$ is the gravity constant.

The right hand side of (14) constitutes the generalized forces of the system, where $F_{x}$,
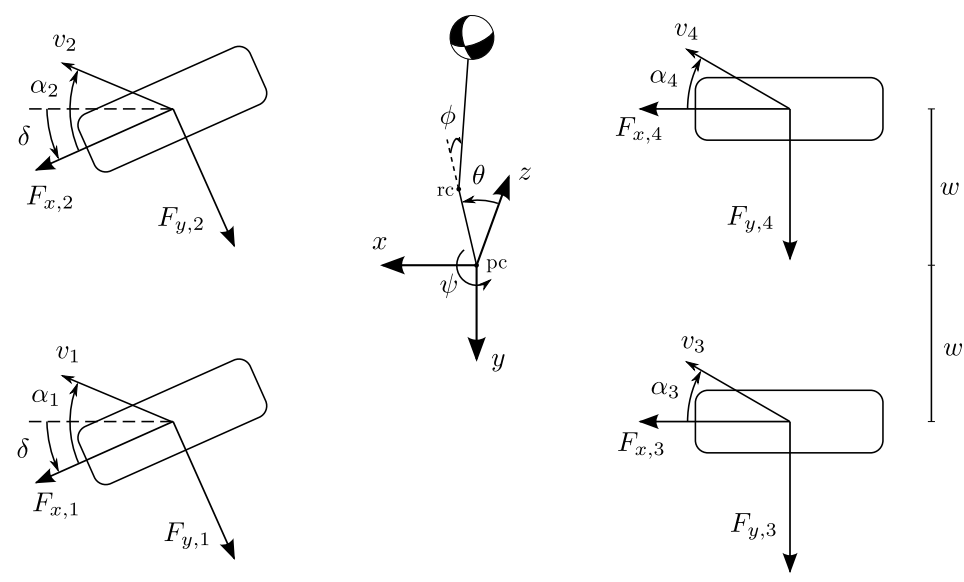

$l_{f}$

$l_{r}$

Figure 5. A sketch of the double-track model, as seen from above. 
$F_{y}$, and $M_{\psi}$ originate from the tire forces and are expressed as

$$
\begin{aligned}
& F_{x}=\left(F_{x, 1}+F_{x, 2}\right) \cos \delta-\left(F_{y, 1}+F_{y, 2}\right) \sin \delta+F_{x, 3}+F_{x, 4}, \\
& F_{y}=\left(F_{y, 1}+F_{y, 2}\right) \cos \delta+\left(F_{x, 1}+F_{x, 2}\right) \sin \delta+F_{y, 3}+F_{y, 4}, \\
& \begin{aligned}
M_{\psi}= & l_{f}\left(\left(F_{y, 1}+F_{y, 2}\right) \cos \delta+\left(F_{x, 1}+F_{x, 2}\right) \sin \delta\right)-l_{r}\left(F_{y, 3}+F_{y, 4}\right)+ \\
& \quad+w\left(\left(-F_{x, 1}+F_{x, 2}\right) \cos \delta+\left(F_{y, 1}-F_{y, 2}\right) \sin \delta+F_{x, 3}+F_{x, 4}\right),
\end{aligned}
\end{aligned}
$$

with $F_{x, i}$ and $F_{y, i}$ representing the longitudinal and lateral tire forces at wheel $i$. The half wheel-track width is denoted $w$, and $l_{f}$ and $l_{r}$ are the distances from the center of gravity to the front and rear axles, respectively.

The maneuvers described in Section 2 are of rollover prone nature with an imminent risk of wheel lift. In this paper, the rollover limit is defined as the instant when both wheels on one side are completely unloaded, which means that when either

$$
F_{z, 1}+F_{z, 3}=0 \quad \text { or } \quad F_{z, 2}+F_{z, 4}=0
$$

the vehicle maneuvering should be constrained by rollover. Note that $F_{z, i}$ here represents the vertical wheel force at wheel $i$, and can only be positive for obvious reasons,

$$
F_{z, i} \geq 0, \quad i \in\{1,2,3,4\}
$$

The general forces in (14c) and (14d), $M_{\phi}$ and $M_{\theta}$, arise from the moments produced by the roll and pitch springs and dampers, determining the load transfer between all wheels, and must therefore account for wheel lift. The pitch moment, $M_{\theta}$, is simply given by

$$
M_{\theta}=K_{\theta} \theta+D_{\theta} \dot{\theta}
$$

with the dynamic front-to-rear load distribution subsequently determined by

$$
\begin{gathered}
F_{z, f}=\frac{m g l_{r}+M_{\theta}}{l_{f}+l_{r}}, \\
F_{z, r}=\frac{m g l_{f}-M_{\theta}}{l_{f}+l_{r}},
\end{gathered}
$$

where $F_{z, f}=F_{z, 1}+F_{z, 2}$ and $F_{z, r}=F_{z, 3}+F_{z, 4}$. The front and rear roll moments are described similarly to (21), but reaches a saturated value when wheel lift occur. The unsaturated front and rear roll moments, denoted $M_{\phi, f}^{*}$ and $M_{\phi, r}^{*}$, are given by

$$
\begin{aligned}
& M_{\phi, f}^{*}=K_{\phi, f} \phi+D_{\phi, f} \dot{\phi}, \\
& M_{\phi, r}^{*}=K_{\phi, r} \phi+D_{\phi, r} \dot{\phi},
\end{aligned}
$$

where $K_{\phi, f}, D_{\phi, f}, K_{\phi, r}$, and $D_{\phi, r}$ are the front- and rear-specific roll stiffness and damping coefficients. The front and rear lateral load transfer is subsequently determined from

$$
\begin{aligned}
& -w\left(F_{z, 1}^{*}-F_{z, 2}^{*}\right)-h_{r c}\left(F_{y, 1}+F_{y, 2}\right)=M_{\phi, f}^{*}, \\
& -w\left(F_{z, 3}^{*}-F_{z, 4}^{*}\right)-h_{r c}\left(F_{y, 3}+F_{y, 4}\right)=M_{\phi, r}^{*} .
\end{aligned}
$$


The saturated normal forces must satisfy $0 \leq F_{z, i} \leq F_{z, f}$ for $i \in\{1,2\}$ and $0 \leq F_{z, i} \leq F_{z, r}$ for $i \in\{3,4\}$. For this purpose, the smooth saturation functions $f_{1, i}$ and $f_{2, i}$ are formulated, using sigmoid functions, according to

$$
\begin{aligned}
F_{z, i} & =F_{z, i}^{*}\left(f_{1, i}-f_{2, i}\right)+F_{z, k} f_{2, i}, \\
f_{1, i} & =\frac{1}{1+e^{-F_{z, i}^{*} \cdot 10^{-3}}}, \\
f_{2, i} & =\frac{1}{1+e^{-\left(F_{z, i}^{*}-F_{z, k}\right) \cdot 10^{-3}}}, \quad i \in\{1,2,3,4\}, \quad k \in\{f, r\} .
\end{aligned}
$$

Note that $k$ here refers to the front and rear axles, where $k=f$ for $i \in\{1,2\}$ and $k=r$ for $i \in\{3,4\}$. The saturated front and rear roll moments, $M_{\phi, f}$ and $M_{\phi, r}$, and the total roll moment, $M_{\phi}$, are finally given by

$$
\begin{aligned}
M_{\phi, f} & =-w\left(F_{z, 1}-F_{z, 2}\right), \\
M_{\phi, r} & =-w\left(F_{z, 3}-F_{z, 4}\right), \\
M_{\phi} & =M_{\phi, f}+M_{\phi, r} .
\end{aligned}
$$

\subsubsection{Wheel and tire dynamics}

The slip angle $\alpha$ and slip ratio $\kappa$ are defined as in [22],

$$
\begin{aligned}
& \dot{\alpha}_{i} \frac{\sigma}{v_{x, i}}+\alpha_{i}=-\arctan \left(\frac{v_{y, i}}{v_{x, i}}\right), \\
& \kappa_{i}=\frac{R_{w} \omega_{i}-v_{x, i}}{v_{x, i}}
\end{aligned}
$$

where $\omega_{i}$ is the wheel angular velocity, $\sigma$ the relaxation length, and $v_{x, i}, v_{y, i}$ are the longitudinal and lateral velocities resolved in the coordinate system of wheel $i$. The wheel rotational dynamics are given by

$$
T_{i}-I_{w} \dot{\omega}_{i}-F_{x, i} R_{w}=0
$$

where $T_{i}$ is the driving or braking torque, $I_{w}$ the wheel inertia, and $R_{w}$ the wheel radius.

The longitudinal and lateral tire forces are computed according to [22]. First, the nominal tire forces $F_{x 0, i}, F_{y 0, i}$ are calculated for pure slip. Subsequently, the final tire forces $F_{x, i}, F_{y, i}$ are determined, taking combined slip in consideration by using the weighting functions $G_{x \alpha, i}$ and $G_{y \kappa, i}$. The complete tire force equations follows from

$$
\begin{aligned}
F_{x 0, i} & =\mu_{x, i} F_{z, i} \sin \left(C_{x, i} \arctan \left(B_{x, i} \kappa_{i}-E_{x, i}\left(B_{x, i} \kappa_{i}-\arctan B_{x, i} \kappa_{i}\right)\right)\right), \\
F_{y 0, i} & =\mu_{y, i} F_{z, i} \sin \left(C_{y, i} \arctan \left(B_{y, i} \alpha_{i}-E_{y, i}\left(B_{y, i} \alpha_{i}-\arctan B_{y, i} \alpha_{i}\right)\right)\right), \\
G_{x \alpha, i} & =\cos \left(C_{x \alpha, i} \arctan \left(B_{x 1, i} \cos \left(\arctan \left(B_{x 2, i} \kappa_{i}\right)\right) \alpha_{i}\right)\right), \\
F_{x, i} & =F_{x 0, i} G_{x \alpha, i}, \\
G_{y \kappa, i} & =\cos \left(C_{y \kappa, i} \arctan \left(B_{y 1, i} \cos \left(\arctan \left(B_{y 2, i} \alpha_{i}\right)\right) \kappa_{i}\right)\right), \\
F_{y, i} & =F_{y 0, i} G_{y \kappa, i} .
\end{aligned}
$$


The vehicle and tire model parameters used in the model equations are listed in Tables 2 and 3 .

\subsection{Planar no-slip model}

The second model is in comparison to the double track model a simplified model. It is a planar model where tire slip is neglected. Hence the name; planar no-slip. It still has the steering angle $\delta$ as input, which now affects the vehicle yaw motions directly. The motion dynamics is described by

$$
\begin{aligned}
\dot{v} & =a_{x}, \\
\dot{\psi} & =\frac{v}{l} \delta,
\end{aligned}
$$

where $v$ is the vehicle velocity, $a_{x}$ the longitudinal acceleration, $\psi$ the yaw angle, and $l=l_{f}+l_{r}$ the wheel base. Since the planar no-slip model has no built-in friction limits, such as the tire model in (29), the friction ellipse is added as a constraint according to

$$
\left(\frac{a_{x}}{\mu_{x} g}\right)^{2}+\left(\frac{a_{y}}{\mu_{y} g}\right)^{2} \leq 1,
$$

where the lateral acceleration $a_{y}$ is determined from

$$
a_{y}=v \dot{\psi}
$$

Similarly, a constraint for rollover has to be introduced. Here, a rigid model is considered, where the lateral load-transfer ratio $L T R$, as described in [23], is defined by

$$
L T R=-\frac{1}{m g}\left(m a_{y} \frac{h_{c g}}{w}\right),
$$

\begin{tabular}{|c|c|}
\hline Notation & Value \\
\hline$\mu_{x}^{*}$ & 0.85 \\
\hline$B_{x}$ & 11.7 \\
\hline$C_{x}$ & 1.69 \\
\hline$E_{x}$ & 0.377 \\
\hline$\mu_{y}^{*}$ & 0.75 \\
\hline$B_{y}$ & 8.86 \\
\hline$C_{y}^{9}$ & 1.19 \\
\hline$E_{y}$ & -1.21 \\
\hline$B_{x 1}$ & 12.4 \\
\hline$B_{x 2}$ & -10.8 \\
\hline$C_{x \alpha}$ & 1.09 \\
\hline$B_{y 1}$ & 6.46 \\
\hline$B_{y 2}$ & 4.20 \\
\hline$C_{y \kappa}$ & 1.08 \\
\hline
\end{tabular}

Table 2. Vehicle model parameters.

\begin{tabular}{ccc}
\hline Notation & Value & Unit \\
\hline$l_{f}$ & 2.45 & $\mathrm{~m}$ \\
$l_{r}$ & 2.55 & $\mathrm{~m}$ \\
$w$ & 1.05 & $\mathrm{~m}$ \\
$m$ & 16200 & $\mathrm{~kg}$ \\
$I_{x x}$ & 24500 & $\mathrm{kgm}^{2}$ \\
$I_{y y}$ & 152800 & $\mathrm{kgm}^{2}$ \\
$I_{z z}$ & 207900 & $\mathrm{kgm}^{2}$ \\
$R_{w}$ & 0.5 & $\mathrm{~m}$ \\
$I_{w}$ & 100 & $\mathrm{kgm}^{2}$ \\
$\sigma$ & 0.5 & $\mathrm{~m}$ \\
$g$ & 9.807 & $\mathrm{~m} / \mathrm{s}^{2}$ \\
$h_{c g}$ & 1.66 & $\mathrm{~m}$ \\
$h_{r c}$ & 0.50 & $\mathrm{~m}$ \\
$K_{\phi, f}, K_{\phi, r}$ & 706 & $\mathrm{kNm} / \mathrm{rad}$ \\
$D_{\phi, f}, D_{\phi, r}$ & 103 & $\mathrm{kNms} / \mathrm{rad}$ \\
$K_{\theta}$ & 2450 & $\mathrm{kNm} / \mathrm{rad}$ \\
$D_{\theta}$ & 1170 & $\mathrm{kNms} / \mathrm{rad}$ \\
\hline
\end{tabular}


and

$$
|L T R| \leq 1
$$

is added as a constraint.

\subsection{Static model}

The last model is a static model where the maximum velocity is determined by the path curvature. This would in many cases be the intuitive first go-to approach when designing a maximum velocity estimator for an on-board application, where the upcoming driving path is known. The equation and constraint for lateral load transfer in (34) and (35) are used in the static model as well. However, the lateral acceleration is determined from the path curvature and vehicle speed, according to

$$
a_{y}=v^{2} C,
$$

where $C$ is the path curvature in (4). The maximum velocity along a given path, according to the static model, can thus be formulated as

$$
v_{\max }(s)=\sqrt{\frac{w g}{C(s) h_{c g}}} .
$$

To assure that the vehicle trajectory does not violate the longitudinal and lateral friction limits, (32) needs to be fulfilled here as well. Using (36), $a_{x}=\frac{\mathrm{d} v}{\mathrm{~d} t}$, and $v=\frac{\mathrm{d} s}{\mathrm{~d} t}$, (32) can be rewritten as a path dependent acceleration constraint, according to

$$
\left|\frac{\mathrm{d} v}{\mathrm{~d} s}\right| \leq \frac{\mu_{x} g}{v(s)} \sqrt{1-\left(\frac{v(s)^{2} C(s)}{\mu_{y} g}\right)^{2}} .
$$

This constraint can be crucial for low-friction surfaces and paths that require severe braking. However, the solutions retrieved with the static model in this paper are unaffected by this constraint.

Notice that, since the static model is dependent on a known path curvature, it is only evaluated for the clothoid turn.

\section{Model analysis}

In this section, some of the transient characteristics of the dynamic models in Section 3 , that is, the double track model and the planar no-slip model, are analyzed to give an indication of which dynamic properties are of most significance. The analysis is limited to studying the frequency response for two important variables with respect to the steer wheel input $\delta$. The first one is the yaw rate $\dot{\psi}$, since the yaw dynamics is one of the most pronounced characteristics in vehicle dynamics. The two models also differ considerably in this aspect, where the planar no-slip model has a linear relation between steer input and yaw rate with yaw inertia completely neglected. The second variable studied is the lateral load-transfer ratio $L T R$. This is an important variable due to the rollover prone 
nature of the considered maneuvers together with the given vehicle configuration, where rollover is expected to limit the vehicle's maneuvering envelop substantially.

The planar no-slip model is, due to its simplicity and already linear form, used as is from Section 3.2. The double track model, however, is highly nonlinear in its original shape, and would be difficult to analyze properly with a simple frequency response analysis if used unmodified. Therefore, it is for this analysis purposely linearized with small angle approximations.

In Figure 6, the yaw rate response for a longitudinal velocity of $v=54 \mathrm{~km} / \mathrm{h}$ is shown. The response of three different models are displayed; the planar no-slip model, and the linearized double-track model with and without relaxation length $\sigma$, that is, the tire slip dynamics in (27a). The relaxation length introduces a tire-force lag, ultimately resulting in a delayed yaw response. Therefore, its effect on the yaw dynamics is here illustrated, in comparison to the effects of only yaw inertia, to evaluate its significance in this respect.

The planar no-slip model will obviously produce a constant response over frequency range due to (31). Both of the linearized double-track models begin to decay from the static value around $0.4 \mathrm{~Hz}$, which can be derived to the yaw inertia $I_{z z}$, a property the planar no-slip model lacks. The relaxation length shows no significant dynamic effect until the excitation frequency reaches $3 \mathrm{~Hz}$, after which it becomes very pronounced.

Figure 7 presents the lateral load-transfer ratio frequency-response for the planar noslip model, together with two variants of the linearized double-track model; with and without roll dynamics. The roll dynamics is here studied due to its direct coupling to the lateral load transfer, see (23) and (24). As for the yaw-rate response, the planar no-slip model has a constant amplitude gain across all frequencies. The double track models begin to deviate from their static values already at $0.1 \mathrm{~Hz}$, due to the effects of yaw inertia and tire slip. At $1.5 \mathrm{~Hz}$, the effects of the roll dynamics are clearly seen by a significant decay in amplitude gain. However, the roll dynamics also contributes to a static offset of $7 \%$ in the amplitude gain. This is due to the lateral displacement of the center of gravity, for a roll angle $|\phi|>0$, where gravity contributes to an added component in the roll moment equation.

With the above analysis in mind, we can expect the double track model to struggle with quick yaw-rate changes, as well as being less constrained by the lateral load-transfer ratio in fast steering-input transients, compared to the planar no-slip model. In addition, the double track model is $7 \%$ more sensitive to lateral load transfer in steady state conditions, which roughly corresponds to a $3.4 \%$ difference in maximum velocity.

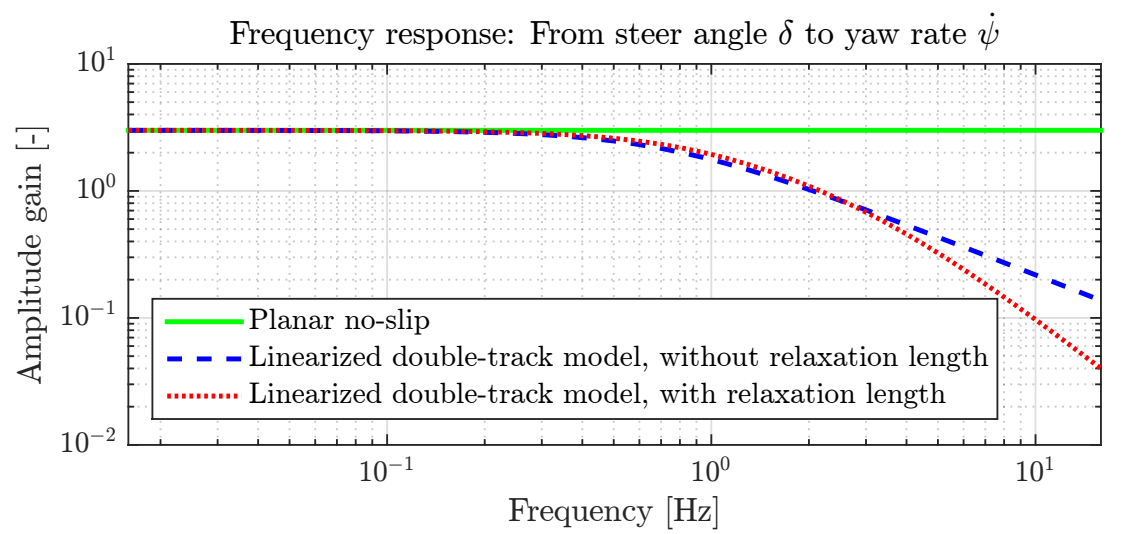

Figure 6. Frequency response diagram for yaw rate $\dot{\psi}$, from the steer wheel input $\delta$, for a velocity of $v=54 \mathrm{~km} / \mathrm{h}$. 


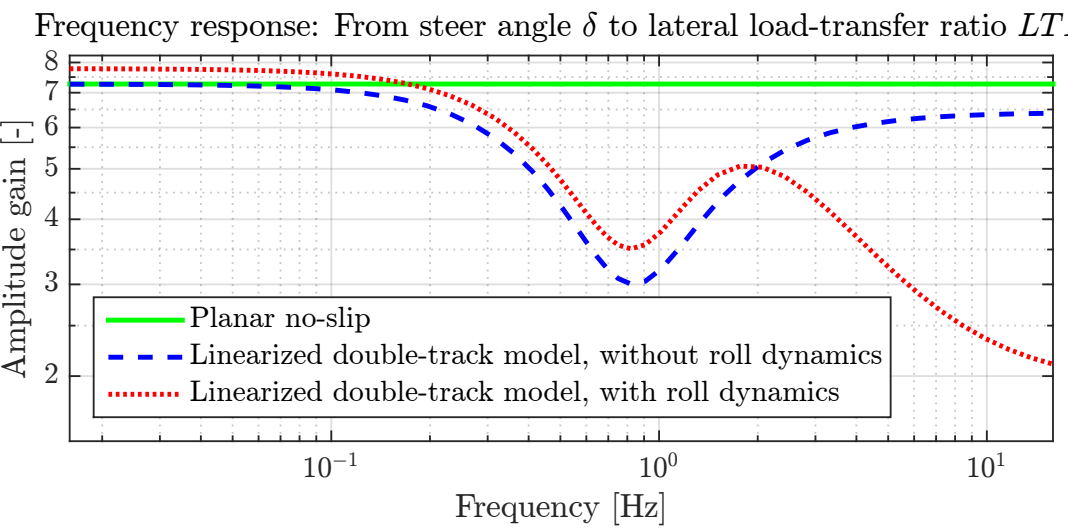

Figure 7. Frequency response diagram for the lateral load-transfer ratio $L T R$, from the steer wheel input $\delta$, for a velocity of $v=54 \mathrm{~km} / \mathrm{h}$.

\section{Optimal control problem formulation}

To obtain the optimal maneuvering solutions for different driving scenarios, a number of optimal control problems are formulated using the maneuver and vehicle models in Sections 2 and 3. Three different maneuvering problems are considered, two for the clothoid turn and one for the double lane change, in which the double track model and the planar no-slip model are employed. The static model, as mentioned in Section 3.3, is only evaluated in the clothoid turn, and due to its simple structure, the optimal velocity profile along the path is acquired from (37).

In the optimal control problem, the vehicle models are formulated as systems of ordinary differential equations, according to

$$
\frac{\mathrm{d} x}{\mathrm{~d} \tau}=f(x(\tau), u(\tau)),
$$

where $x$ represent the model state, $u$ the control input, and $f$ the model dynamics. The optimization problems, and thereby also the models, are discretized over the distance along the path $s$ for the clothoid turn, and over time $t$ for the double lane change. Hence, $\tau$ here represents the discretization variable, where $\tau \in\{s, t\}$. Note that the path dynamics, in (6)-(8), is included in the state vector $x$ when $s$ is used as the discretization variable.

The control input for the double track model consist of the steering rate $\dot{\delta}$ and the braking/driving torque $T$ for each wheel, resulting in

$$
u=\left[\dot{\delta}, T_{1}, T_{2}, T_{3}, T_{4}\right]^{T} .
$$

The planar no-slip model lacks wheel dynamics. Instead, the longitudinal acceleration $a_{x}$ is used directly as a control input, together with $\dot{\delta}$ according to

$$
u=\left[\dot{\delta}, a_{x}\right]^{T} .
$$

The steering angle and steering rate is, for both models, constrained by $|\delta| \leq \delta_{\max }$ and $|\dot{\delta}| \leq \dot{\delta}_{\max }$, with $\delta_{\max }=0.5 \mathrm{rad}$ and $\dot{\delta}_{\max }=1 \mathrm{rad} / \mathrm{s}$. The wheel torque limits, according to

$$
T_{\min } \leq T_{i} \leq T_{\max , i}, \quad i \in\{1,2,3,4\},
$$


are $T_{\min }=-\mu_{x} m g, T_{\max , 1}=T_{\max , 2}=0 \mathrm{Nm}$, and $T_{\max , 3}=T_{\max , 4}=T_{\max }=13.4 \mathrm{kNm}$. The longitudinal acceleration, for the planar no-slip model, is constrained according to $a_{x, \min } \leq a_{x} \leq a_{x, \max }$, where $a_{x, \min }=\mu_{x} m g$ and $a_{x, \max }=T_{\max } \frac{R_{w}}{m}$. In addition, the wheel rotational velocities, for the double track model, are limited to be positive only, $\omega_{i} \geq 0$.

The optimal control problem to be solved can then be formulated according to

$$
\begin{aligned}
\text { minimize } & J \\
\text { subject to } & \frac{\mathrm{d} x}{\mathrm{~d} \tau}=f(x(\tau), u(\tau)), \\
& g(x(\tau), u(\tau)) \leq 0, \\
& x(0)=x_{0}, \quad x\left(\tau_{f}\right)=x_{f}, \quad \tau \in\left[0, \tau_{f}\right],
\end{aligned}
$$

where $J$ is the optimization objective, $g$ the continuous constraints, $x_{0}$ and $x_{f}$ the initial and terminal constraints, and $\tau_{f}$ is the final distance $s_{f}$ or final time $t_{f}$.

Below follows the maneuver specific preferences, objectives, and constraints for the clothoid turn and the double lane change.

\subsection{Clothoid turn}

The clothoid turn is described by the curvilinear abscissa $s$, along which the curvature $C$ is described, see Section 2.1. The path tolerance $e_{\max }$ defines the path boundaries within which the vehicle must stay. This is assured by adding (9) to the constraints in $g$.

The clothoid turn is subject for two different problems; constant speed and braking in turn, which are described separately below.

\subsubsection{Constant speed}

The objective of the constant speed problem is to find the maximum constant velocity the vehicle can drive through the clothoid turn. The optimization objective is formulated according to

$$
J=-v_{i n i t}+\int_{0}^{s_{f}} u^{T} Q u \mathrm{~d} s
$$

where $v_{\text {init }}$ is the initial velocity. A small cost on $u$ is introduced with the integral part, to reduce excessive control inputs, with the diagonal weight matrix

$$
Q=\frac{N_{e}}{s_{f}} \operatorname{diag}\left(\eta_{1}, \eta_{2} \frac{1}{R_{w} m}, \eta_{2} \frac{1}{R_{w} m}, \eta_{2} \frac{1}{R_{w} m}\right) \quad \text { and } \quad Q=\frac{N_{e}}{s_{f}} \operatorname{diag}\left(\eta_{1}, \eta_{2}\right)
$$

for the double track model and the planar no-slip model, respectively. Here, $N_{e}$ is the number of discretization elements, and the parameters $\eta_{1}=0.01$ and $\eta_{2}=0.01$ are used to balance the cost of the control inputs in the objective function. For the double track model, the wheel torques are normalized with $\frac{1}{R_{w} m}$, making the sum of them comparable with $a_{x}$ in the planar no-slip model.

For the planar no-slip model, the velocity is constrained to be constant, $v \equiv v_{\text {init }}$, by constraining the acceleration to be zero, $a_{x} \equiv 0$. The double track model has more force components acting in the longitudinal direction as well as a lateral component of the velocity vector. Thus, constraining the velocity to be exactly zero can be unnecessary 
restrictive for the solver, why a small velocity slack of $v_{t o l}= \pm 0.05 \mathrm{~km} / \mathrm{h}$ has been introduced for the double track model, according to

$$
\left|v-v_{\text {init }}\right| \leq \Delta v_{t o l} .
$$

The maximum speed for the static model is simply computed with (37) at the minimum curve radius $R_{\min }$.

\subsubsection{Braking in turn}

In this problem, the vehicle enters the turn with excessive speed and the objective is to find a solution where the vehicle starts decelerating as late as possible and not more than necessary. This is achieved with a minimum time formulation, with the cost function

$$
J=t_{f}+\int_{0}^{s_{f}} u^{T} Q u \mathrm{~d} s,
$$

where $t_{f}$ is the final time. The weight matrices of (41) are also used here, however, with $\eta_{2}=10^{-5}$ to not restrict the vehicle from large braking inputs. The initial velocity $v_{\text {init }}$ is here proportional to $\sqrt{R_{\min }}$, according to

$$
v_{\text {init }}=1.5 \sqrt{\frac{R_{\min } w}{h_{c g} g}},
$$

which is equivalent to $50 \%$ above the maximum speed predicted by the static model at a turn radius of $R_{\text {min }}$.

Only the braking performance is of interest in this problem, which might be somewhat distorted if the vehicle is allowed to accelerate as well. To prevent this, all wheel torques are constrained to negative values $T_{i} \leq 0$, for the double track model, and the longitudinal acceleration $a_{x} \leq 0$ for the planar no-slip model.

\subsection{Double lane change}

The double lane-change maneuver is, unlike the clothoid turn, not described along a predefined path. Instead, the maneuvering space constraints are described in the global Cartesian coordinate-system $X Y$. The outermost boundaries in Figure 4 are described with the constraints

$$
0 \leq X \leq L_{D L C} \quad \text { and } \quad 0 \leq Y \leq W_{D L C}
$$

where $(X, Y)$ is the vehicle position. The obstacles are described using superellipses according to

$$
\left(\frac{X-d_{x, i}}{R_{x, i}}\right)^{6}+\left(\frac{Y-d_{x, i}}{R_{y, i}}\right)^{6} \geq 1
$$

where $d_{x, i}, d_{y, i}$ defines the origin of the ellipse, and $R_{x, i}, R_{y, i}$ the corresponding height and width. The index $i$ here refers to the individual obstacles; Obstacle 1, 2, and 3, in Figure 4. 
In the double lane-change problem, the objective is to find out how fast the vehicle can negotiate the avoidance maneuver. Thus, a minimum-time formulation is used, with the cost function

$$
J=t_{f}+\int_{0}^{t_{f}} u^{T} Q u \mathrm{~d} t
$$

Similar to the clothoid turn problems, a small control input penalty is added, to reduce excessive control inputs, using a weight matrix similar to (41), according to

$$
Q=\frac{N_{e}}{t_{f}} \operatorname{diag}\left(\eta_{1}, \eta_{2} \frac{1}{R_{w} m}, \eta_{2} \frac{1}{R_{w} m}, \eta_{2} \frac{1}{R_{w} m}\right) \quad \text { and } \quad Q=\frac{N_{e}}{t_{f}} \operatorname{diag}\left(\eta_{1}, \eta_{2}\right),
$$

for the double track model and the planar no-slip model. Here, $\eta_{1}=0.01$ and $\eta_{2}=0.01$ is used.

\subsection{Solving the optimal control problem}

Due to the complex problem formulation resulting in non-trivial solutions, the optimal control problems were solved with numerical methods. The continuous optimalcontrol problem in (39) is discretized over the distance horizon $s \in\left[0, s_{f}\right]$ or time horizon $t \in\left[0, t_{f}\right]$, depending on the considered problem, into $N_{e}=200$ elements of equal size. Within each element, the state dynamics is approximated with a fourth order polynomial using $N_{p}=3$ collocation points per element, distributed according to Radau collocation [5]. Each element is assigned a set of control variables that are constant over the entire element interval.

The discretized optimal-control problem is implemented in Python using the syntax and tools provided by the CasADi framework [1], which automatically generates symbolic expression and derivatives necessary for the solver. The resulting nonlinear program is subsequently solved with the general purpose nonlinear-programming solver Ipopt [26], using the linear solver HSL MA57 [14].

\subsubsection{Initialization procedure}

Providing the solver with a proper initial guess is often an essential prerequisite to retrieve the optimal solution to complex optimal-control problems, such as those considered here. To generate these initial guesses, a simulation approach similar to the initialization procedure in [3] was used. The vehicle models were simulated using a PI-controller based driver-model following a predefined path. This path is already given on beforehand for the clothoid turn problems, but for the double lane-change problems a path negotiating the maneuver and avoiding the obstacles was purposely constructed.

\subsubsection{Solver statistics}

The effort required by the solver to find the optimal solution varies somewhat for the different problems, maneuver properties, and vehicle models used. The median values of the solver's solution times and number of iterations are listed in Table 4, for the double track model and the planar no-slip model when applied to the problems specified in Sections 5.1 and 5.2. The median is shown here due to the solver struggling with a few number of problem configurations, resulting in much longer solution times, which skew the mean value figures. All of the problems were solved with a standard PC equipped 
with an Intel Core i3 processor. It is evident from the tabular data that the planar no-slip model is easier to handle for the solver, especially for the clothoid turn problems. For the double lane change it is still superior in terms of solution time, however, require noticeable number of additional iterations on average. In addition, the planar no-slip model requires 200-300 MB of memory by the solver, compared to 5-8 GB for the double track model.

Table 4. Median values of the solver's solution times and number of iterations for the double track model and the planar no-slip model, when applied to the clothoid turn problems and the double lane-change problem.

\begin{tabular}{ccccc}
\hline & \multicolumn{2}{c}{ Median solution time, $[\mathrm{s}]$} & \multicolumn{2}{c}{ Median number of iterations, [-] } \\
& Double track & Planar no slip & Double track & Planar no slip \\
\hline $\begin{array}{c}\text { Clothoid turn, } \\
\text { constant speed }\end{array}$ & 108.8 & 2.7 & 46.5 & 25 \\
\hline $\begin{array}{c}\text { Clothoid turn, } \\
\text { braking in turn }\end{array}$ & 132.7 & 3.3 & 82.5 & 41.5 \\
\hline Double lane change & 186.9 & 8.7 & 122 & 187 \\
\hline
\end{tabular}

\section{Results}

In this section, the results obtained by solving the optimal control problems, described in Section 5, is presented when applied to the maneuvers and vehicle models in Sections 2 and 3. The results are presented in three main subsections referring to constant speed in clothoid turn, to braking in clothoid turn, and to double lane change. For each main subsection there is a description of the studies performed, a presentation of a typical individual maneuver, a parametric study where tolerances and characteristics have been varied, and finally some comments on the implications. An overall discussion of the results is then provided in Section 7.

To distinguish between variables associated with specific vehicle models, the following notation is introduced:

- DT: Double track model.

- PNS: Planar no-slip model.

- Stat: Static model.

For example, $v_{D T}$ refers to the velocity of the double track model.

\subsection{Clothoid turn - Constant speed}

The results for the clothoid turn maneuver, described in Section 2.1, are presented. The results for the two different optimization formulations constant speed and braking in turn, described in Section 5.1, have been obtained and the constant speed is presented here, whereas the case of braking is presented in Section 6.2 below. In both cases, results from all three models are presented, where the solutions to the double track model and the planar no-slip model were obtained with the optimization procedure in Section 5, while the results for the static model was computed from (34)-(37).

The solutions has been obtained for multiple clothoid geometries, by varying the minimum radius $R_{\min }$ and the maximum path-curvature rate $\left(\frac{\mathrm{d} C}{\mathrm{~d} s}\right)_{\max }$, according to

$$
R_{\text {min }} \in\{15,20,25,30,40,50\} \mathrm{m},
$$


and

$$
\left(\frac{\mathrm{d} C}{\mathrm{~d} s}\right)_{\max } \in\{0.3,0.5,1,1.5,2,2.5,3\} \cdot 10^{-3} \mathrm{~m}^{-2}
$$

In addition, the maximum path deviation $e_{\max }$ was varied with the following thresholds,

$$
e_{\max } \in\{1,5,10,20,40,80\} \mathrm{cm}
$$

The objective in this first problem configuration is to find the maximum speed the vehicle can drive through the clothoid turn, with the velocity constrained to being constant throughout the whole maneuver. The results are here presented in two batches. In the first, the minimum path radius $R_{\min }$ and the maximum path deviation $e_{\max }$ are varied. The different $R_{\min }$ should here scale the clothoid path uniformly. Hence, the maximum path-curvature rate will vary with $R_{\min }$ according to

$$
\left(\frac{\mathrm{d} C}{\mathrm{~d} s}\right)_{\max }=\frac{1}{2 R_{\min }^{2}}
$$

where $\Delta s$ in (10) has been substituted for $2 R_{\min }$. The combinations of different $R_{\min }$ and $e_{\max }$ results in 36 solutions for each model. In the second batch, the maximum path-curvature rate $\left(\frac{\mathrm{d} C}{\mathrm{~d} s}\right)_{\max }$ and the maximum path deviation $e_{\max }$ are varied, while the minimum radius is kept at $R_{\min }=30 \mathrm{~m}$. This results in 42 solutions per model. Note that, since the static model lacks the freedom of deviating from the path and its maximum velocity is determined solely by the path curvature (or, in this case, the minimum radius), it only has six unique solutions for the first batch, and one for the second. The results corresponding to these two batches are separately presented below, preceded by an analysis of one individual maneuver configuration.

\subsubsection{Clothoid turn - Constant speed: Individual maneuver}

As a typical example of the clothoid turn maneuver with constant speed, the result for $e_{\max }=5 \mathrm{~cm}, R_{\min }=30 \mathrm{~m}$, and $\left(\frac{\mathrm{d} C}{\mathrm{~d} s}\right)_{\max }=\frac{1}{2 R_{\min }^{2}}=0.56 \cdot 10^{-3} \mathrm{~m}^{-2}$ is shown in detail in Figure 8 . The steer angle $\delta$, velocity $v$, lateral acceleration $a_{y}$, yaw rate $\dot{\psi}$, path deviation $e$, and lateral load-transfer ratio $L T R$ are shown over the driven path distance $s$. The double track model and the planar no-slip model clearly utilize the allowed path deviation $e$ to straighten out the tightest section of the clothoid turn. See, for example, the flat regions in $a_{y}$ and $L T R$ around $s=90 \mathrm{~m}$. Otherwise, the planar no-slip model utilize the path space more, while the double track model spend most of the time near the outer path boundary.

Since the maneuver is smooth and with a slowly varying steering input, characteristics similar to steady state can in some terms be expected. Thus, a $7 \%$ difference in maximum lateral acceleration between the simple models and the double track model would be presumed, as anticipated by the linear model analysis in Section 4 . However, the maximum acceleration for the planar no-slip model and the static model is $a_{y, \max }^{P N S}=a_{y, \max }^{\text {Stat }}=6.19$ $\mathrm{m} / \mathrm{s}^{2}$, and for the double track model $a_{y, \max }^{D T}=5.63 \mathrm{~m} / \mathrm{s}^{2}$. This corresponds to a $9 \%$ difference, indicating that other properties and characteristics than the displacement of the center of gravity indirectly constrains the double track model, such as a weakened roll stiffness when wheel lift occur. 
The planar no-slip model is at its $a_{y, \max }$ for the longest, which is also where it is constrained by the lateral load-transfer ratio. The double track model is unable to reach the same performance in this aspect due to the added dynamics, such as the yaw dynamics, contributing to a slower yaw acceleration. See the inclination of the yaw rate $\dot{\psi}$ in Figure 8.

\subsubsection{Clothoid turn - Constant speed: Maneuver parameter study for minimum radius and path deviation tolerance}

In Table 5, the obtained maximum constant velocity for each model is listed, over different $R_{\min }$ and $e_{\max }$ (which is the first batch described above). This data is also presented as surface plots in Figure 9(a), where the maximum velocity has been normalized with the square root of the minimum radius, that is,

$$
\frac{v_{\max }}{\sqrt{R_{\min }}}
$$

to ease the visual comparison. Note that the static model here forms a horizontal plane as a result of this. As expected, all models show an increased velocity for an increased minimum radius. The same yields for an increased path deviation threshold for the double track model and the planar no-slip model, since they can reduce the effective curvature by cutting the corner. In Figure 9(b), the surfaces of the double track model and the static model from Figure 9(a) are shown, viewed from above. The line wherein these surfaces intersect marks the boundary for which of the models that predicts the highest velocity. To the left, in the gray area, the static model predicts the highest velocity, and vice versa in the turquoise area.

Figure 10 displays the relative velocity difference $\Delta v$ between the double track model

Clothoid turn: Constant speed, $R_{\min }=30 \mathrm{~m}, e_{\max }=5 \mathrm{~cm}$.
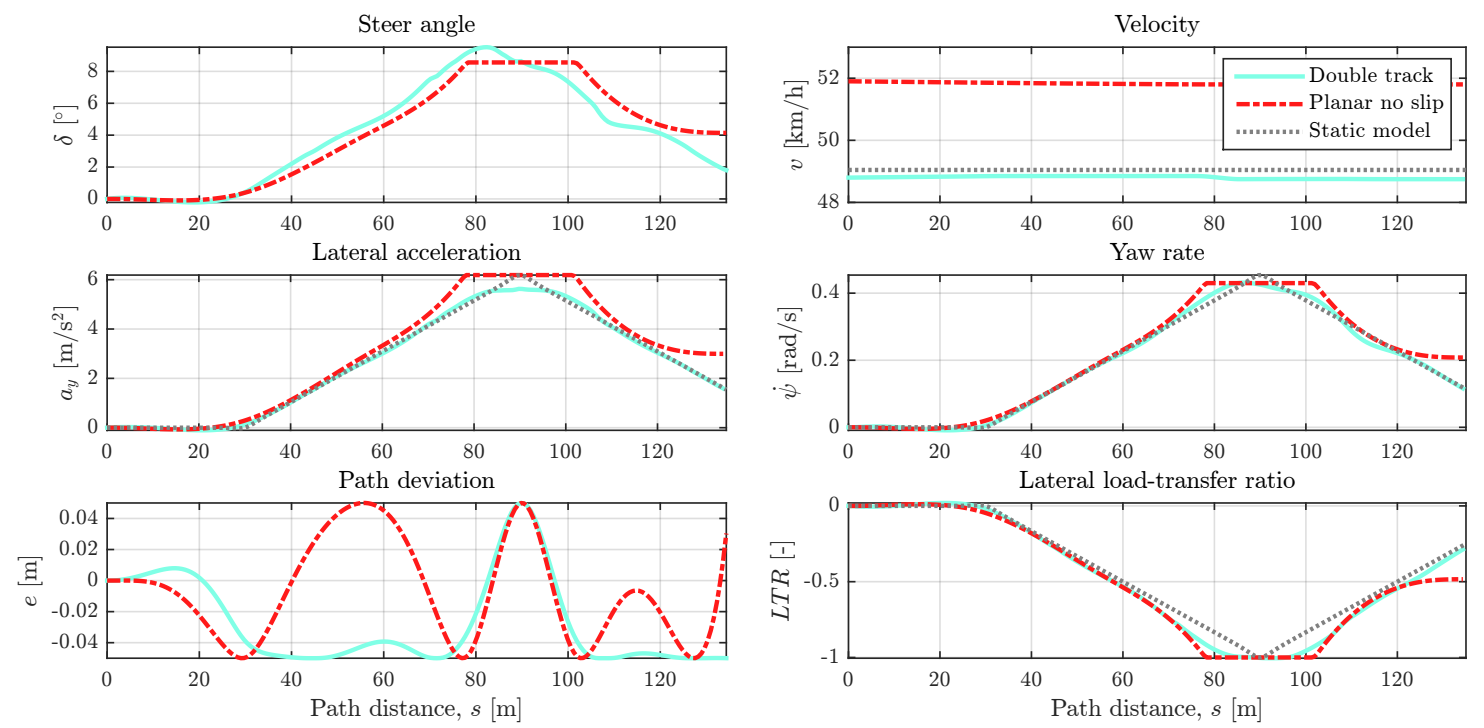

Figure 8. The optimal solutions for all three models in the clothoid maneuver, with a minimum radius of $R_{m i n}=$ $30 \mathrm{~m}$ and a maximum path deviation of $e_{\max }=5 \mathrm{~cm}$. The objective is to obtain the maximum velocity through the maneuver, where the velocity is constrained to be constant. 


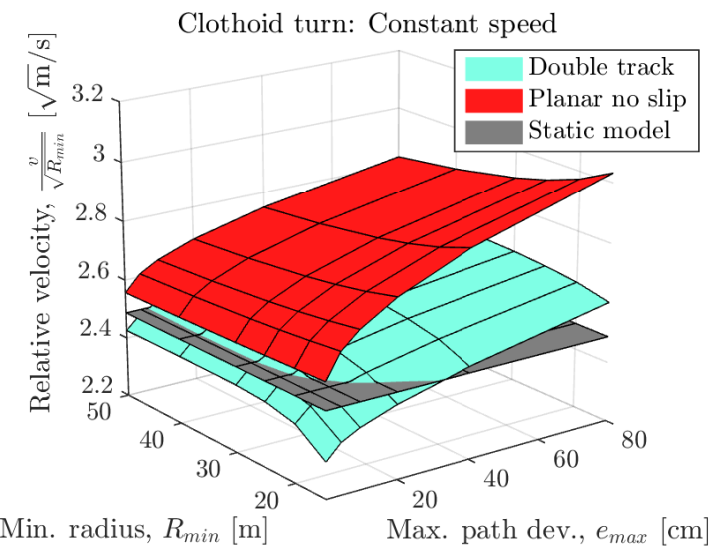

(a) The relative maximum speed for all three models.

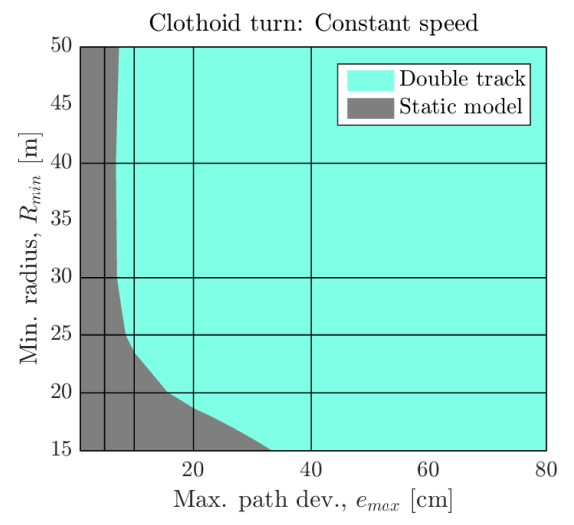

(b) The relative maximum speed for the double track model and the static model, viewed from above.

Figure 9. The maximum constant speed $v_{\max }$, normalized by the square root of the minimum path radius $\sqrt{R_{\min }}$, obtained for different $R_{\min }$ and $e_{\max }$.

and the planar no-slip model, defined by

$$
\Delta v=\frac{v_{P N S}-v_{D T}}{v_{P N S}}
$$

Here it is evident that the difference in obtained maximum speed increases substantially as the minimum radius drops off. This is primarily due to the yaw dynamics of the double track model, where the yaw inertia in combination with tire forces limited by the road friction, introduces a yaw-rate time lag. This lag is more prominent for tighter maneuvers, where higher yaw acceleration is necessary. Figure 11 shows the portion of the maneuver where the lateral load-transfer ratio is an active constraint, that is, when $|L T R|=1$. Here, it is evident that the double track model in fact is constrained by something other than the load transfer in the tighter maneuvers. The planar no-slip model do not

Table 5. Maximum constant speed obtained for the clothoid turn. Results are shown for all three models over different maximum allowed path deviations $e_{\max }$ and minimum path radii $R_{\min }$.

\begin{tabular}{lccccccc}
\hline \multicolumn{5}{c}{ Clothoid turn: Maximum constant velocity, $v_{\max }[\mathrm{km} / \mathrm{h}]$} \\
\hline \multirow{2}{*}{ Model } & $\begin{array}{c}\text { Max. path dev., } \\
e_{\max }[\mathrm{cm}]\end{array}$ & 15 & 20 & 25 & 30 & 40 & 50 \\
\hline Double & 1 & 32.4 & 38.6 & 43.5 & 47.8 & 55.3 & 61.8 \\
track & 5 & 33.1 & 39.3 & 44.4 & 48.8 & 56.4 & 63.0 \\
& 10 & 33.5 & 39.7 & 44.9 & 49.4 & 57.1 & 63.7 \\
& 20 & 34.1 & 40.3 & 45.5 & 50.2 & 58.0 & 64.7 \\
& 40 & 35.0 & 41.1 & 46.4 & 51.1 & 59.1 & 66.0 \\
& 80 & 36.3 & 42.3 & 47.5 & 52.2 & 60.5 & 67.6 \\
\hline Planar & 1 & 36.0 & 41.5 & 46.3 & 50.6 & 58.3 & 65.1 \\
no slip & 5 & 37.1 & 42.7 & 47.5 & 51.9 & 59.6 & 66.4 \\
& 10 & 37.9 & 43.5 & 48.3 & 52.7 & 60.5 & 67.3 \\
& 20 & 38.9 & 44.5 & 49.4 & 53.8 & 61.6 & 68.5 \\
& 40 & 40.3 & 45.9 & 50.8 & 55.3 & 63.1 & 70.0 \\
& 80 & 42.3 & 47.9 & 52.8 & 57.2 & 65.1 & 72.0 \\
\hline Static & - & 34.7 & 40.0 & 44.8 & 49.0 & 56.6 & 63.3 \\
\hline
\end{tabular}


change its $L T R$ limited portion much across the different $R_{\text {min }}$, for most path deviation thresholds, indicating that these solutions are similar and only geometrically scaled in proportion to $R_{\min }$. However, results obtained for $e_{\max }=80 \mathrm{~cm}$ slightly contradicts this. Since the path deviation limit is relatively larger, in relation to the path geometry, at $R_{\text {min }}=15 \mathrm{~m}$ compared to $R_{\text {min }}=50 \mathrm{~m}$, a large $e_{\max }$ can affect the results for the planar no-slip model also.

\subsubsection{Clothoid turn - Constant speed: Maneuver parameter study for curvature rate and path deviation tolerance}

The second batch for the clothoid turn with constant speed contains results from different maximum allowed path deviations $e_{\max }$ and maximum path-curvature change rates $\left(\frac{\mathrm{d} C}{\mathrm{~d} s}\right)_{\max }$. Table 6 presents the obtained maximum speed for all three vehicle models. Note that the static model returns the same velocity for all $\left(\frac{\mathrm{d} C}{\mathrm{~d} s}\right)_{\max }$ values, since $R_{\text {min }}=30 \mathrm{~m}$ for all cases here. The maximum velocities for the double track model and the planar no-slip model are also visualized as surfaces in Figures 12(a) and 12(b), where both models show similar tendencies. The obtained maximum speed increases for larger $e_{\max }$, which is reasonable, but also for larger $\left(\frac{\mathrm{d} C}{\mathrm{~d} s}\right)_{\max }$ when $e_{\max } \leq 5 \mathrm{~cm}$, which is slightly counter intuitive. Since large $\left(\frac{\mathrm{d} C}{\mathrm{~d} s}\right)_{\max }$ results in shorter clothoid paths having less of a sweeping bend, see Figure 3(b), the vehicle models can straighten out these maneuvers noticeably compared to the original path curvature. For $e_{\max }=1 \mathrm{~cm}$, however, the maximum speed decreases as $\left(\frac{\mathrm{d} C}{\mathrm{~d} s}\right)_{\text {max }}$ increases for the double track model. This is explained by the higher path-curvature change rate requiring faster yaw motions, with the yaw inertia's effects limiting the vehicle motions more, thus forcing vehicle velocity to be reduced. The planar no-slip model does not experience this due to the absence of proper yaw dynamics. The two models differ at least by $5.2 \%$ in obtained maximum velocity, for $\left(\frac{\mathrm{d} C}{\mathrm{~d} s}\right)_{\max }=0.3 \cdot 10^{-3} \mathrm{~m}^{2}$ and $e_{\max }=1 \mathrm{~cm}$. This corresponds to a narrow path with slowly varying curvature, where the yaw properties have minor impact on the overall dynamics.

In Figure 13, the maximum velocity of the double track model and the static model

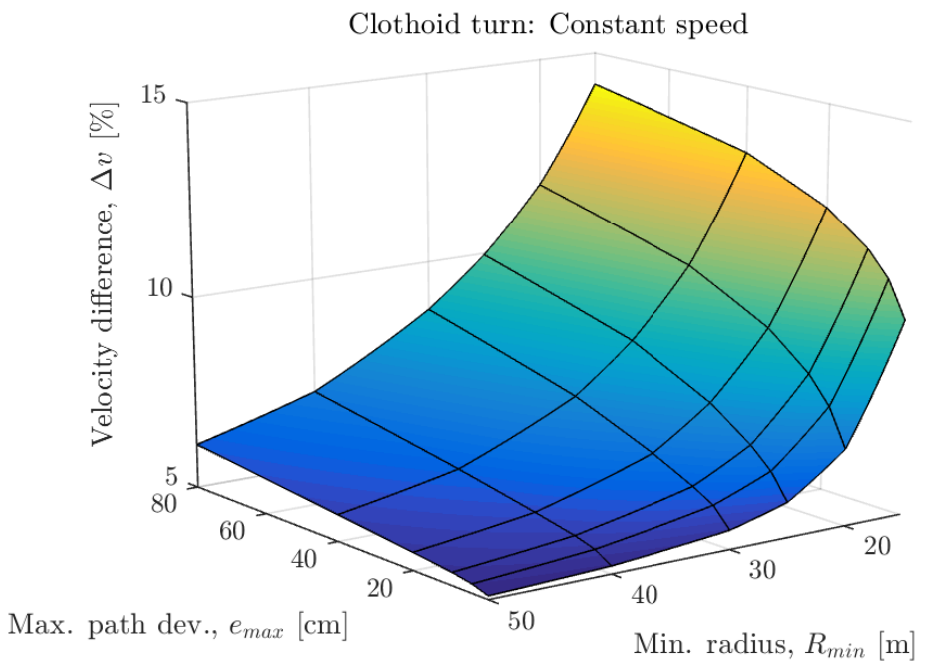

Figure 10. Relative difference of the obtained maximum speed between the double track model and the planar no-slip model, for various $R_{\min }$ and $e_{\max }$. 


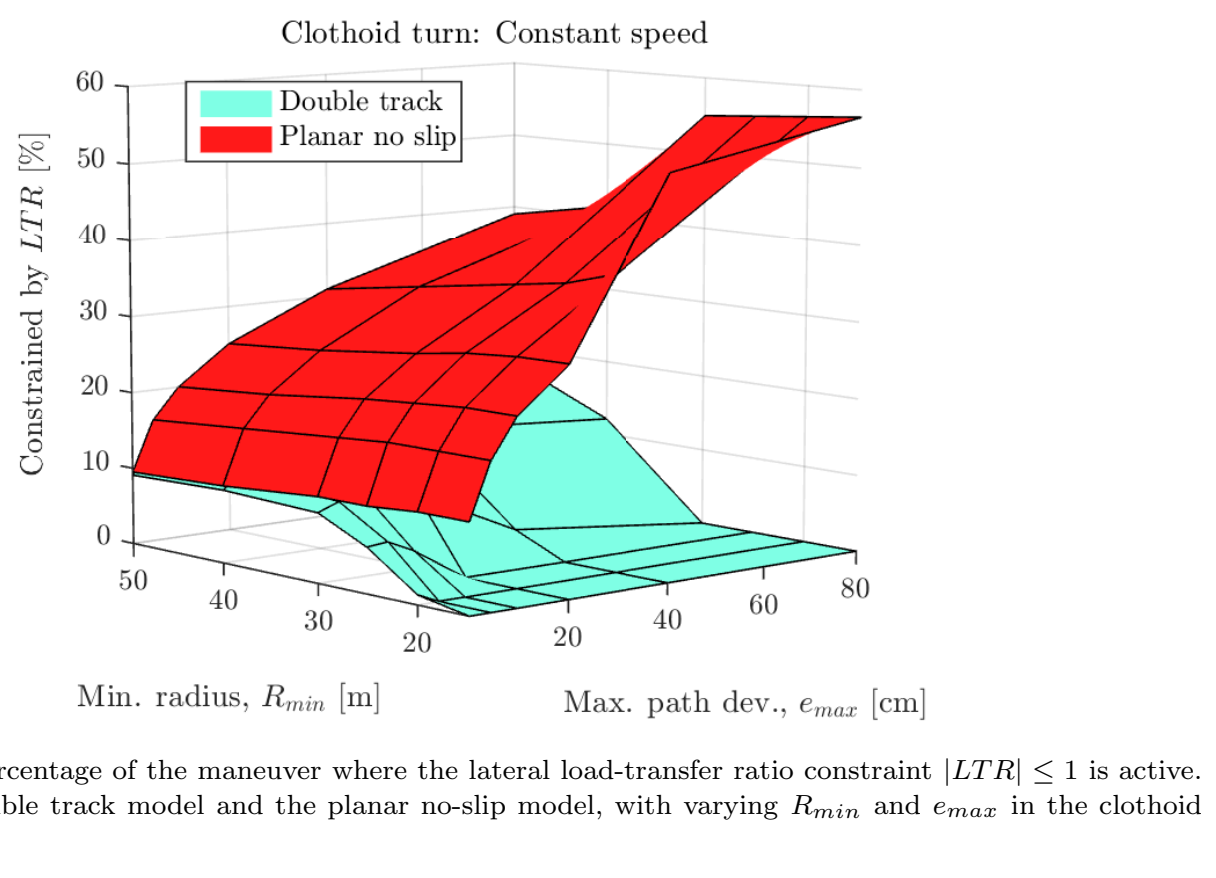

Min. radius, $R_{\min }[\mathrm{m}] \quad$ Max. path dev., $e_{\max }[\mathrm{cm}]$

Figure 11. The percentage of the maneuver where the lateral load-transfer ratio constraint $|L T R| \leq 1$ is active. Results for the double track model and the planar no-slip model, with varying $R_{\min }$ and $e_{\max }$ in the clothoid turn, are shown.

are shown, similar to Figure 9(b). The line, where the two surfaces intersects, follows $e_{\max }=5 \mathrm{~cm}$ fairly consistently. For slow path-curvature change rates, the total curve length increases and the gain from cutting the corner is reduced. The vehicle can therefore not benefit from a specific path tolerance $e_{\max }$ to the same extent. Hence, the line in question will curve to the right, in Figure 13, for small $\left(\frac{\mathrm{d} C}{\mathrm{~d} s}\right)_{\max }$.

Table 6. Maximum constant speed obtained for the clothoid turn. Results are shown for all three models over different maximum allowed path deviations $e_{\max }$ and maximum path-curvature change rate $\left(\frac{\mathrm{d} C}{\mathrm{~d} s}\right)_{\max }$, while the minimum path radius is retained at $R_{\min }=30 \mathrm{~m}$.

\begin{tabular}{cccccccccc}
\hline \multicolumn{7}{c}{ Clothoid turn: Maximum constant velocity, $v_{\max }[\mathrm{km} / \mathrm{h}]$} \\
\hline \multirow{2}{*}{ Model } & Max. path dev., & \multicolumn{7}{c}{ Max. path curvature rate, $\left(\frac{\mathrm{d} C}{\mathrm{~d} s}\right)_{\max }\left[10^{-3} \mathrm{~m}^{-2}\right]$} \\
& $e_{\max }[\mathrm{cm}]$ & 0.3 & 0.5 & 1 & 1.5 & 2 & 2.5 & 3 \\
\hline Double & 1 & 47.5 & 47.8 & 48.1 & 47.0 & 47.4 & 44.8 & 43.6 \\
track & 5 & 48.2 & 48.7 & 49.6 & 49.2 & 50.4 & 49.3 & 49.3 \\
& 10 & 48.6 & 49.3 & 50.6 & 50.9 & 52.8 & 52.7 & 53.8 \\
& 20 & 49.1 & 49.9 & 52.0 & 53.3 & 56.5 & 58.3 & 62.1 \\
& 40 & 49.6 & 50.7 & 53.9 & 57.1 & 63.5 & 70.4 & 81.9 \\
& 80 & 50.1 & 51.7 & 57.1 & 65.2 & 77.5 & 91.1 & 118.0 \\
\hline Planar & 1 & 50.1 & 50.5 & 51.3 & 50.5 & 52.5 & 51.5 & 51.9 \\
no slip & 5 & 50.9 & 51.7 & 53.3 & 53.2 & 56.1 & 55.8 & 57.1 \\
& 10 & 51.4 & 52.5 & 54.7 & 55.2 & 58.8 & 59.1 & 61.3 \\
& 20 & 52.1 & 53.5 & 56.6 & 57.9 & 62.7 & 64.8 & 69.8 \\
& 40 & 52.9 & 54.8 & 59.2 & 62.1 & 70.4 & 77.8 & 91.4 \\
& 80 & 54.0 & 56.5 & 63.1 & 70.7 & 89.3 & 108.1 & 144.6 \\
\hline Static & - & 49.0 & 49.0 & 49.0 & 49.0 & 49.0 & 49.0 & 49.0 \\
\hline
\end{tabular}

(n) 


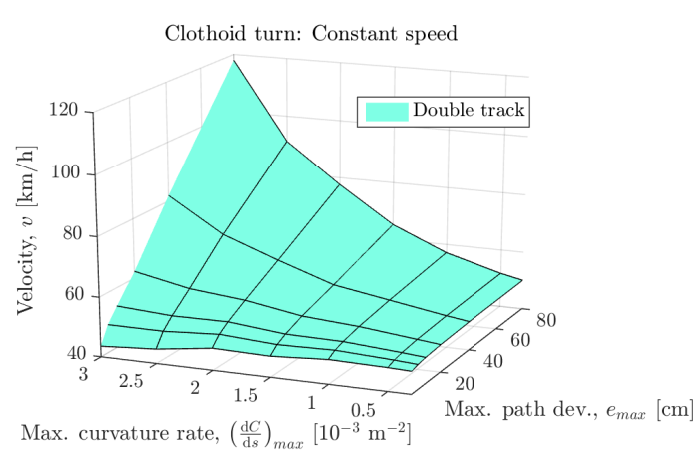

(a) Maximum speed for the double track model.

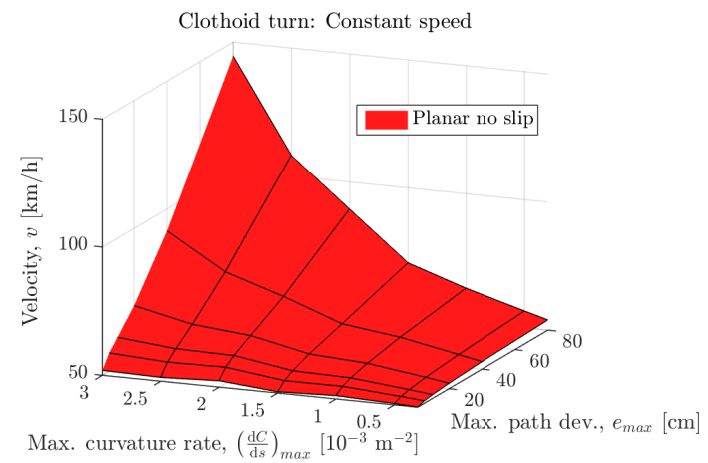

(b) Maximum speed for the planar no-slip model.

Figure 12. The maximum constant speed $v_{\max }$ obtained for different $\left(\frac{\mathrm{d} C}{\mathrm{~d} s}\right)_{\max }$ and $e_{\max }$.

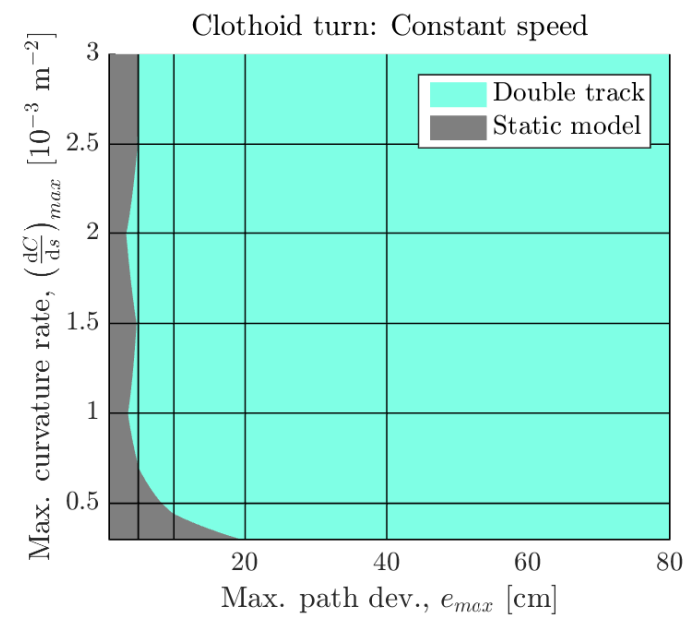

Figure 13. The maximum speed $v_{\max }$ obtained for the double track model and the static model, viewed from above, for various $\left(\frac{\mathrm{d} C}{\mathrm{~d} s}\right)_{\max }$ and $e_{\max }$, similar to Figure 9(b). Areas covered by the double track models surface, in this view, represents the region where this model obtains a higher speed than the static model.

\subsubsection{Clothoid turn - Constant speed: Some observations and implications}

Based on the results, it is interesting to give some speculations related to the original problem of path following in autonomous driving. One question is what tolerance is needed for a path tracking to be rather simple. Here Figures 9(b) and 13 gives an answer based on model complexity. These figures display a clear path-tolerance limit, almost independent of path geometry, which indicates the task is simple: A static model can be sufficient if the tolerance is $7 \mathrm{~cm}$ or $5 \mathrm{~cm}$, respectively. However, in the lower left corner of the figures, the maneuvers become more aggressive. Thus, the inertia of the vehicle comes into play and a tolerance with path geometry dependence is needed.

Figure 10 shows the relative difference in velocity between the double track model and the much simpler planar no-slip model. It can be seen that the difference is between 5 and $15 \%$. This indicates that the simple and computationally more effective model (that is, the planar no-slip model) can be used in combination with a safety margin. However, to obtain close to optimal performance, this margin should not be fixed. Figure 10 can here be used as a look-up table for the required margin. 

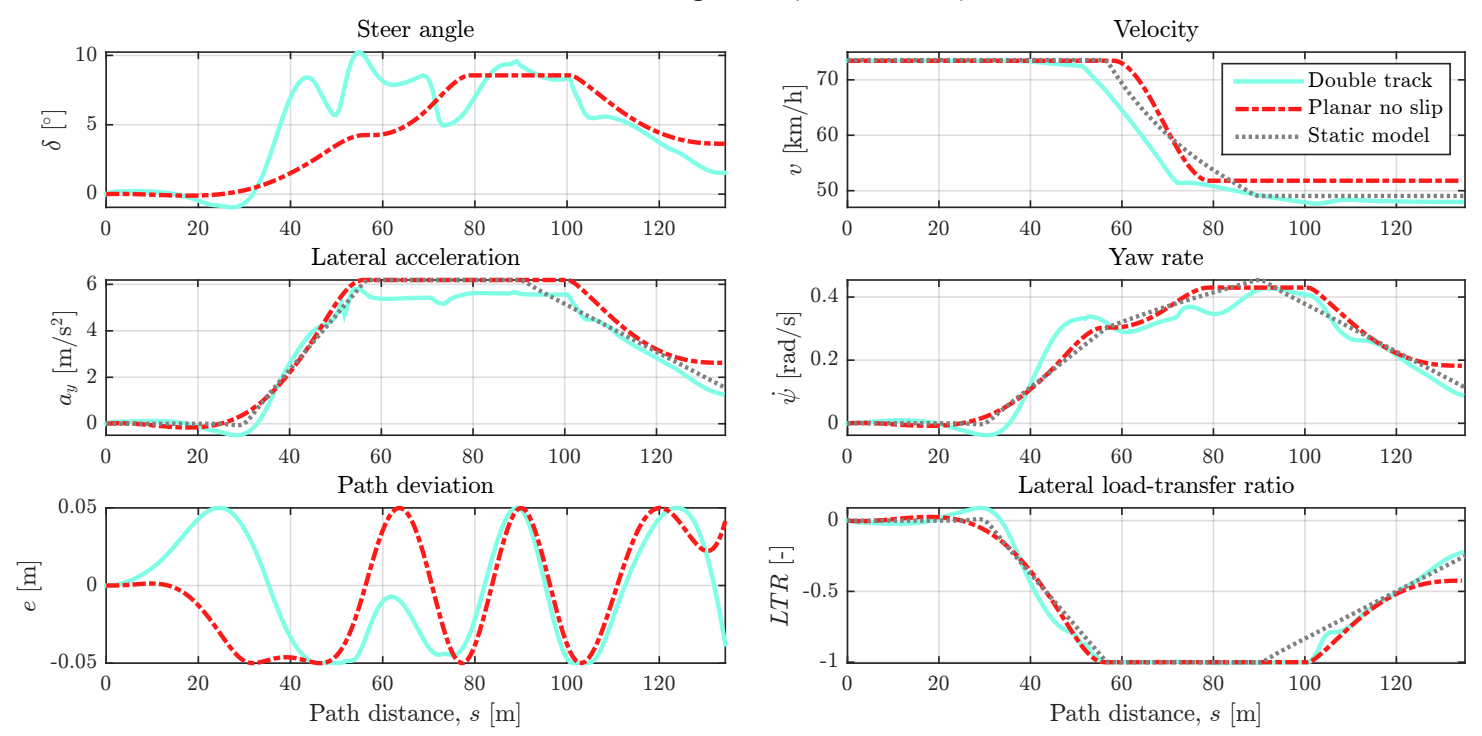

Figure 14. The optimal solutions for all three models in the clothoid maneuver, with a minimum radius of $R_{\min }=30 \mathrm{~m}$ and a maximum path deviation of $e_{\max }=5 \mathrm{~cm}$. The objective is to drive as quickly as possible through the turn without accelerating.

\subsection{Clothoid turn - Braking in turn}

In this maneuver, the vehicle approach the clothoid turn with excessive speed, and the objective is to drive as quickly as possible through the turn without accelerating. Thus, the vehicle is forced to reduce the speed and initiate the braking phase as late as possible to minimize the time loss.

Results obtained from different path deviation thresholds $e_{\max }$ and minimum path radii $R_{\min }$ are presented below, starting with an analysis of an individual maneuver configuration.

\subsubsection{Clothoid turn - Braking in turn: Individual maneuver}

Figure 14 presents the obtained optimal solutions for all three models, with $R_{\min }=30 \mathrm{~m}$ and $e_{\max }=5 \mathrm{~cm}$ as a typical example of this maneuver. The velocity profile $v$ here clearly indicates that the initial speed is too high, and has to be significantly reduced before the critical point of the path, at $s=90 \mathrm{~m}$, where the minimum radius $R_{\text {min }}$ appears. The double track model has to initiate the braking phase the earliest. The planar no-slip model is the last to initiate the braking phase, decelerates the most, and maintain the highest speed after braking. Compared to the static model, these features derive from the planar no-slip model's freedom to deviate from the path. The double track model exploits this as well. However, it is unable to handle lateral accelerations of the same amplitudes, is slightly constrained by the yaw dynamics, and has to reduce the speed more and earlier in the maneuver.

For the double track model, a significant part of the braking is performed when $L T R=-1$, implying both of the left wheels are completely unloaded. Thus, braking forces can only be generated on the right side wheels, which in turn will create a yaw moment counteracting the yaw motion required by the path. This is seen in Figure 14, where the double track model rapidly increases the yaw rate $\dot{\psi}$ in the beginning, and subsequently levels out with a slight drop off when the braking is initiated at $s=51 \mathrm{~m}$. 


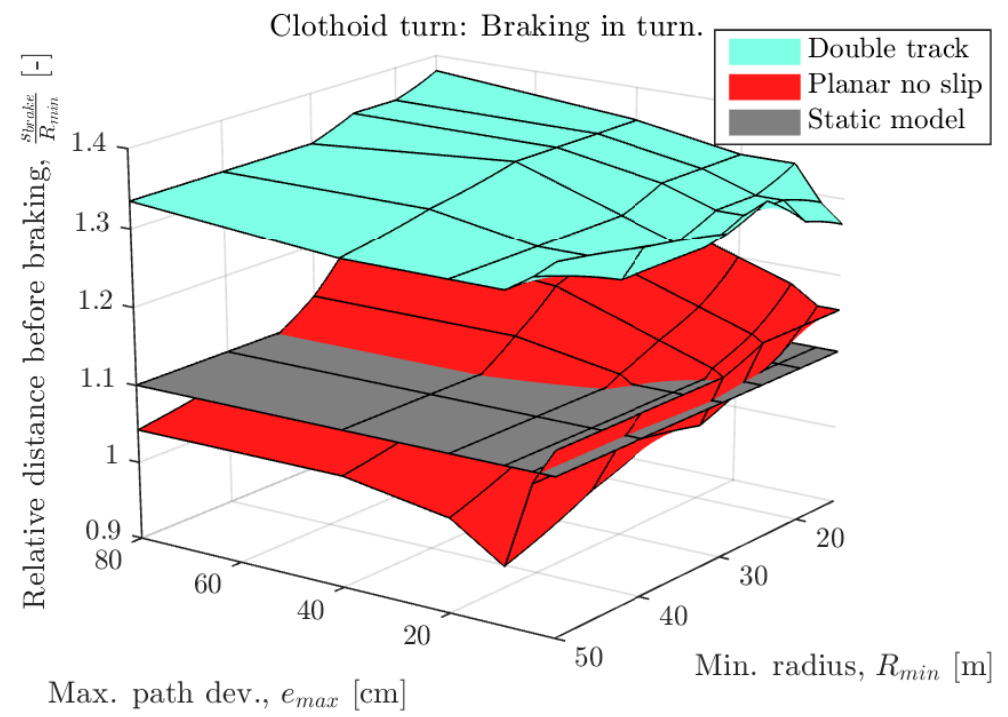

Figure 15. The distance along the path between the start of the braking phase and the path's minimum-radius, $s_{\text {braking }}$, normalized over the specific $R_{\min }$ for each maneuver configuration. Results corresponding to all three vehicle models, for different $R_{\min }$ and $e_{\max }$, are visualized.

\subsubsection{Clothoid turn - Braking in turn: Maneuver parameter study}

The distance between the start of the braking phase and the $s_{2}$ mark in Figure 2 is here denoted $s_{\text {brake }}$. This is a measure of how close to the clothoid path minimum-radius the vehicle can go before braking needs to be initiated. To execute the maneuver in a time optimal manner, it is beneficial to brake late and quickly, that is, a small sbrake contributes to a high mean velocity. In Figure 15, the distance $s_{\text {brake }}$, normalized over the minimum path radius $R_{\text {min }}$, is shown for all three vehicle models over different $R_{\min }$ and $e_{\max }$. Note that the velocity profile from the static model is identical for all variations, only scaled proportional to the clothoid geometry, resulting the horizontal plane in Figure 15. The double track model also sees relatively consistent results, in this aspect, over the various $R_{\min }$ and $e_{\max }$, varying within $9.6 \%$. However, on average, it has to start braking $21.2 \%$ earlier compared to the static model. The planar no-slip model experiences a considerably more scattered results, varying $24.4 \%$ between the shortest and longest relative distances in Figure 15.

The time duration under braking $t_{\text {braking }}$ is for the static model proportional to $\sqrt{R_{\text {min }}}$, since

$$
t_{\text {braking }}=\frac{s_{\text {braking }}}{v_{\text {init }}-v_{\max }\left(s_{2}\right)},
$$

where $s_{\text {braking }}$ is the distance traveled during braking (which is proportional to $R_{\text {min }}$ ) and $v_{\max }\left(s_{2}\right)$ is the maximum speed according to (37) at $s=s_{2}$, which is the location of the minimum radius along the path. Thus, $v_{\text {init }}-v_{\max }\left(s_{2}\right)$ accounts for the total speed reduction for the static model in the maneuver and is proportional to $\sqrt{R_{\min }}$. Figure 16 shows $t_{\text {braking }}$ normalized over $\sqrt{R_{\text {min }}}$, where the result associated with the static model therefore assumes the shape of a horizontal plane. Both the double track model and the planar no-slip model experience an increased braking duration for small path deviation thresholds, due to the limited maneuvering space. As $e_{\max } \rightarrow 0$, it is reasonable for these models to approach the similar behavior as the static model. For $e_{\max }>5 \mathrm{~cm}$, the 


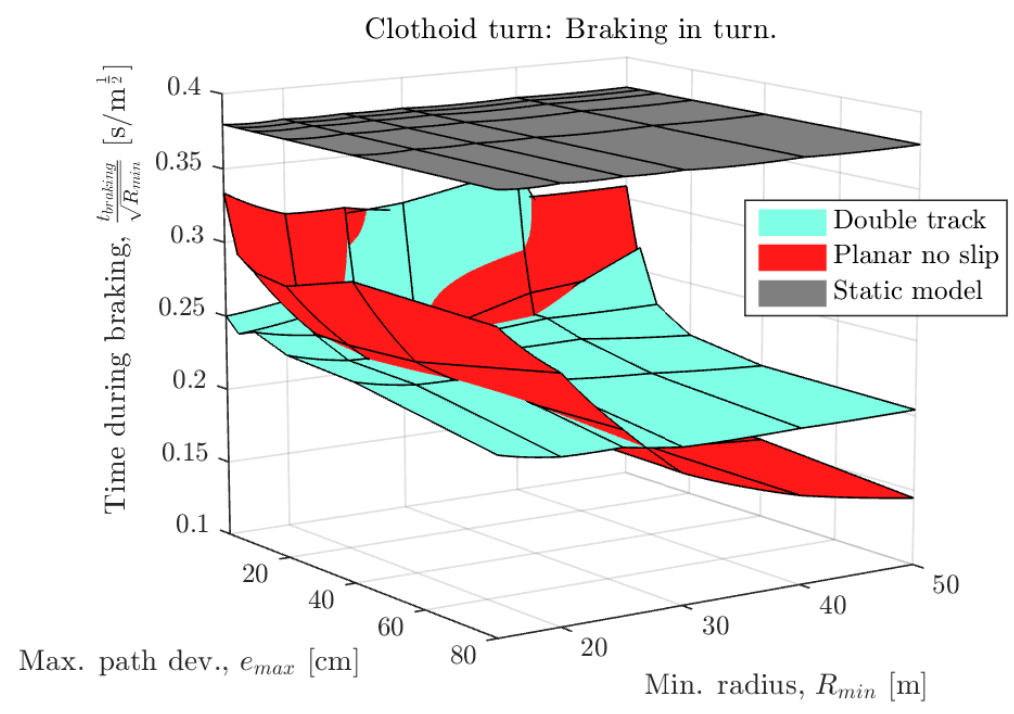

Figure 16. The braking time $t_{\text {braking }}$ for all three models, normalized over $\sqrt{R_{\min }}$, in the clothoid turn for various $R_{\min }$ and $e_{\max }$.

double track model show consistent results, where the braking duration time is roughly proportional to $\sqrt{R_{\min }}$ regardless of size or narrowness of the maneuver. The planar noslip model clearly displays braking strategies that differ over different $R_{\text {min }}$, but seems rather steady for various path deviation thresholds $e_{\max }>5 \mathrm{~cm}$.

\subsubsection{Clothoid turn - Braking in turn: Some observations and implications}

The most interesting aspects of the results above, is the braking characteristics shown by the double track model, and in particular, its similarities to the static model. The results shown in Figures 15 and 16 indicate that the braking strategy for the double track model can be approximated with a scaled version of the static model. This is, from a computational point of view, promising for on-board systems.

\subsection{Double lane change}

Here the results associated with the double lane-change maneuver, described in Section 2.2, is presented. Optimal solutions obtained for the double track model and the planar no-slip model, with the methodology in Section 5, are shown and discussed. The maneuver properties of the double lane change is varied by changing the width of the obstacles $w_{o b}$, according to

$$
w_{o b} \in\{2,4,6,8\} \mathrm{m},
$$

and scaling the road friction by

$$
\lambda_{\mu} \in\{0.4,0.6,0.8,1.0,1.2\},
$$

as described in Section 2.2 . 


\subsubsection{Double lane change: Individual maneuver}

The solutions obtained for an obstacle width of $w_{o b}=4 \mathrm{~m}$ and a friction scaling of $\lambda_{\mu}=1.0$ is presented in Figure 17, exemplifying a typical double lane-change maneuver. Here, the double track model clearly requires more work with the steering input $\delta$ to control the yaw motions, and cannot attain the desired yaw rate as quickly as the planar no-slip model, due to its yaw inertia. Hence, this is constraining the double track model, preventing it to reach the limit set by the lateral load transfer $(|L T R|=1)$. For the planar no-slip model, however, this constraint is in practice active throughout the whole maneuver. Therefore, the planar no-slip model can also maintain a higher velocity, on average $23 \%$ higher in this maneuver.

The uppermost plot in Figure 17 show the global coordinates for the two models, where the solid black lines define the maneuver boundaries within the vehicle must operate, and the obstacles that must be avoided. The lateral path difference $e_{Y}$ describes the offset in the $Y$ direction between the paths of the double track model and the planar no-slip model, according to

$$
e_{Y}=Y_{D T}-Y_{P N S}
$$

In this respect, the two paths differ at most by $\left|e_{Y}\right|=0.25 \mathrm{~m}$ at $X=24 \mathrm{~m}$. The path curvature $C$ and the path curvature rate $\frac{\mathrm{d} C}{\mathrm{~d} s}$, referring to the paths driven by the two models, have quite different appearances. The planar no-slip experience a piece-wise constant curvature, a result of exploiting $|L T R|=1$ to such an extent. The double track model is unable to generate path change-rates $\frac{\mathrm{d} C}{\mathrm{~d} s}$ of the same magnitude, and thus has to compensate with larger peak values of $C$.

\subsubsection{Double lane change: Maneuver parameter study}

Table 7 presents the mean velocity $v_{\text {mean }}$ obtained for the double track model and the planar no-slip model, in the double lane-change maneuver, for various obstacle widths $w_{o b}$ and friction scaling $\lambda_{\mu}$. The optimization objective is here to minimize the total time of the maneuver, which implies $v_{\text {mean }}$ is to be maximized. The mean velocity is decreasing for wider obstacles, and increasing for higher friction. However, between $\lambda_{\mu}=0.8$ and $\lambda_{\mu}=1.0$ the mean velocity is seen to stagnate. This is particularly evident for the planar no-slip model, which in practice has two different constraint on the lateral acceleration; one determined by the lateral load transfer, deriving from (34) and (35),

$$
a_{y}^{L T R}=\frac{w}{h_{c g}} g
$$

and the other constituted by the available friction,

$$
a_{y}^{\mu}=\mu_{y} g=\lambda_{\mu} \mu_{y}^{*} g
$$

derived from (32) when $a_{x}=0$. Since both $\left|a_{y}\right| \leq a_{y}^{L T R}$ and $\left|a_{y}\right| \leq a_{y}^{\mu}$ must yield, only one of these constraints will act as an active constraint, depending on the available friction. The switch will occur when $a_{y}^{L T R}=a_{y}^{\mu}$, which is equivalent to a friction scaling of $\lambda_{\mu}=0.84$.

Figure 19 presents the relative difference of the mean velocity between the two models, 

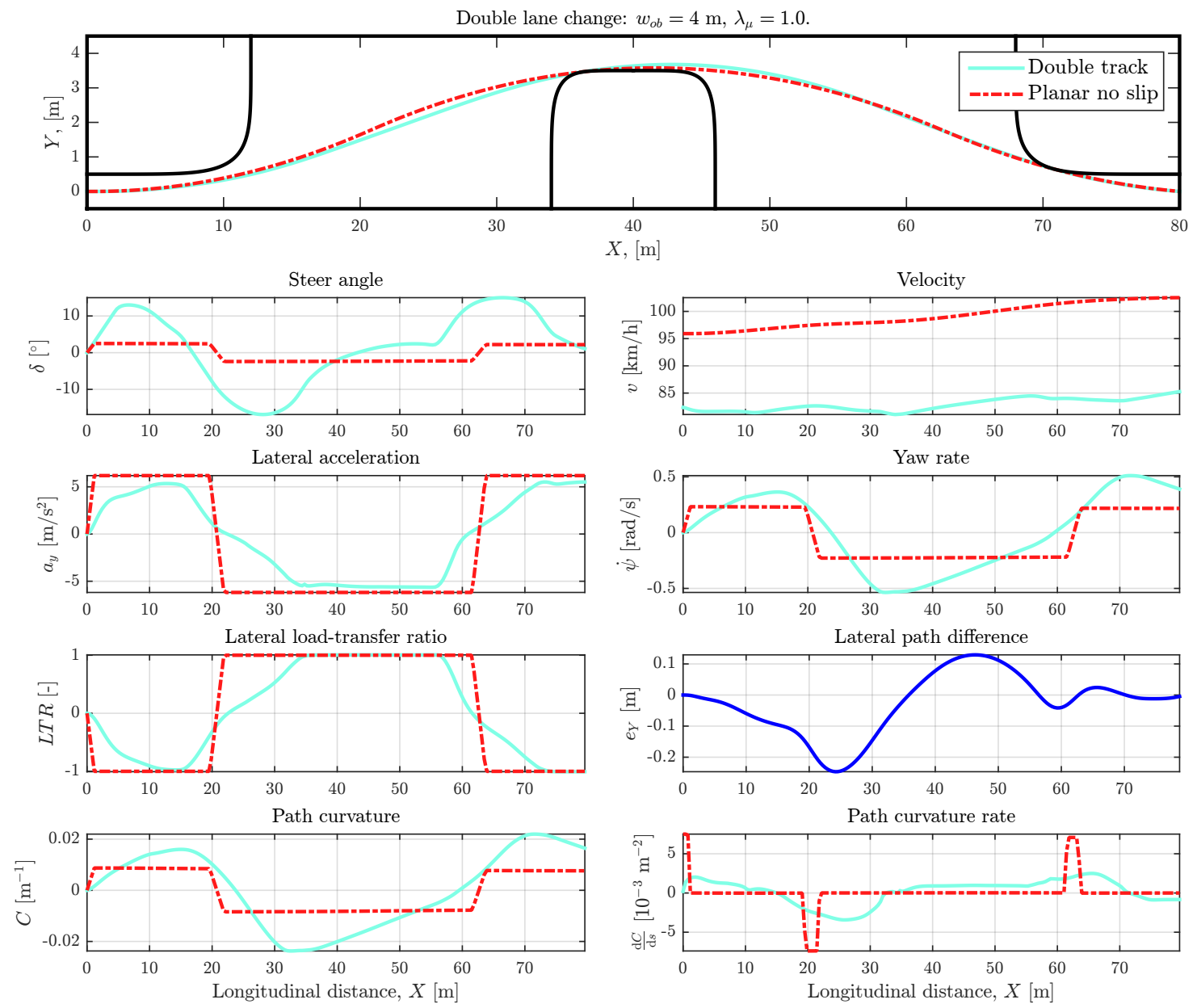

Figure 17. The optimal solutions for the double track model and the planar no-slip model in the double lanechange maneuver, where the obstacle width is $w_{o b}=4 \mathrm{~m}$ and the friction scaling is $\lambda_{\mu}=1.0$. The objective is formalized as a minimum-time problem, with the initial velocity as a free parameter. Note that $e_{Y}$ refers to the difference between the paths of the two models.

that is, the velocities listed in Table 7 , and is defined as

$$
\Delta v_{\text {mean }}=\frac{v_{\text {mean }}^{P N S}-v_{\text {mean }}^{D T}}{v_{\text {mean }}^{P N S}}
$$

The percentage of the maneuver where the lateral load-transfer constraint is active is shown in Figure 18 for each of the two models. For example, with an obstacle width of $w_{o b}=2 \mathrm{~m}$ and a friction scaling of $\lambda_{\mu}=1.2$, the double track model experiences $|L T R|=1$ during $22 \%$ of the total time. Since the planar no-slip model switches from being limited by friction to being limited by the lateral load transfer at $\lambda_{\mu}=0.84$, it goes from $0 \%$ to $94 \%$ after $\lambda_{\mu}=0.8$ in Figure 18. For small friction scaling coefficients of $\lambda_{\mu} \leq 0.6$, the double track model is also seen to never reach $|L T R|=1$. However, the percentage increases with both higher $\lambda_{\mu}$ and larger $w_{o b}$. The former is due to the increased available tire forces, which enables quicker yaw handling. The latter is explained by the wider obstacle causing the maneuver to be less dynamic, where the vehicle endure more time in relatively stable cornering, while the narrow obstacle forces the vehicle to spend more of the maneuvering time in yaw transients. The double track model will suffer 
from this, but the planar no-slip model will not (due to its lack of yaw dynamics). For the same reason, the velocity difference $\Delta v_{\text {mean }}$, in Figure 19, increases for smaller $w_{o b}$. In Figure 19, a distinct peak appears along $\lambda_{\mu}=0.8$, which is related to the discussion regarding $a_{y}^{L T R}$ and $a_{y}^{\mu}$ above. The planar no-slip model attains its maximum speed at $\lambda_{\mu}=0.84$, while the double track model can benefit from a higher friction by being able to generate larger tire forces, that results in faster yaw motions which ultimately enables the vehicle to reach $|L T R|=1$ quicker.

Table 7. The mean velocity for the double track model and the planar no-slip model in the double lane-change maneuver. Results are presented for different obstacle widths $w_{o b}$ and differently scaled friction $\lambda_{\mu}$.

\begin{tabular}{ccccccc}
\hline \multicolumn{5}{c}{ Double lane change: Mean velocity, $v_{\text {mean }}[\mathrm{km} / \mathrm{h}]$} \\
\hline Model & $\begin{array}{c}\text { Obstacle width, } \\
w_{\text {ob }}[\mathrm{m}]\end{array}$ & 0.4 & 0.6 & 0.8 & 1.0 & 1.2 \\
\hline Double & 2 & 73.7 & 88.0 & 97.9 & 106.6 & 112.9 \\
track & 4 & 55.6 & 67.2 & 76.5 & 83.2 & 85.9 \\
& 6 & 48.0 & 57.9 & 66.0 & 71.3 & 72.8 \\
& 8 & 43.1 & 52.0 & 59.3 & 63.6 & 65.0 \\
\hline Planar & 2 & 96.5 & 117.8 & 135.8 & 139.3 & 139.3 \\
no slip & 4 & 69.1 & 84.1 & 96.7 & 99.4 & 99.4 \\
& 6 & 57.2 & 69.5 & 79.8 & 82.2 & 82.2 \\
& 8 & 50.4 & 61.1 & 70.0 & 72.2 & 72.2 \\
\hline
\end{tabular}

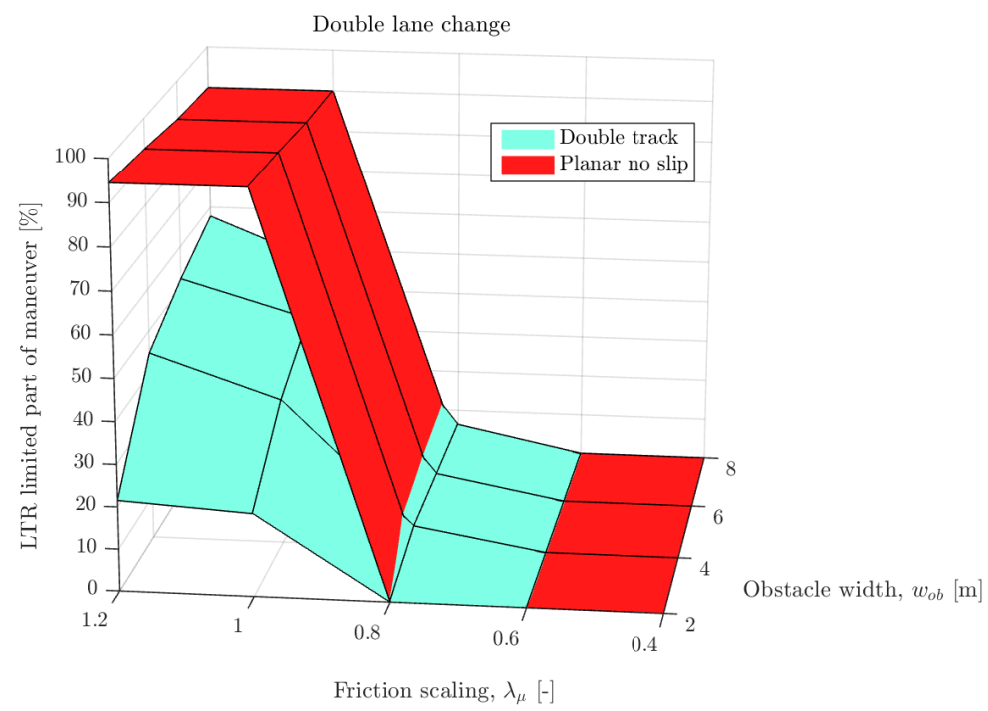

Figure 18. Percentage of the double lane-change maneuver where the lateral load-transfer constraint is active, that is, when $|L T R|=1$.

The lateral path difference $e_{Y}$, in (46), is a measure of how similar the paths of the two models are. In Figure 20, the maximum value of this path difference, according to $e_{Y, \max }=\max \left|e_{Y}\right|$, is shown over various $w_{o b}$ and $\lambda_{\mu}$. Here, $e_{Y, \max }$ is seen to increase for wider obstacles, approximately proportional to the obstacle width $w_{o b}$. For example, at $\lambda_{\mu}=0.8$, the maximum path difference is $e_{Y, \max }=0.12 \mathrm{~m}$ for $w_{o b}=2 \mathrm{~m}$ and $e_{Y, \max }=0.51 \mathrm{~m}$ for $w_{o b}=8 \mathrm{~m}$, meaning $e_{Y, \max }$ is proportionally increasing with larger 
$w_{o b}$. The path difference also increases for lower friction, which is particularly visible for $w_{o b}=8 \mathrm{~m}$, since the double track model struggles more with initiating the yaw motion and turning when the available friction is reduced.

The maximum values of the path curvature $C$ and the path curvature rate $\frac{\mathrm{d} C}{\mathrm{~d} s}$ indicate the two models capabilities in terms of navigating through the confined space stipulated by the double lane-change maneuver. The maximum values of these quantities, $C_{\max }$ and $\left(\frac{\mathrm{d} C}{\mathrm{~d} s}\right)_{\max }$, for the double track model and the planar no-slip model are shown in Figure 21. The planar no-slip model is, in terms of path curvature, only limited by the

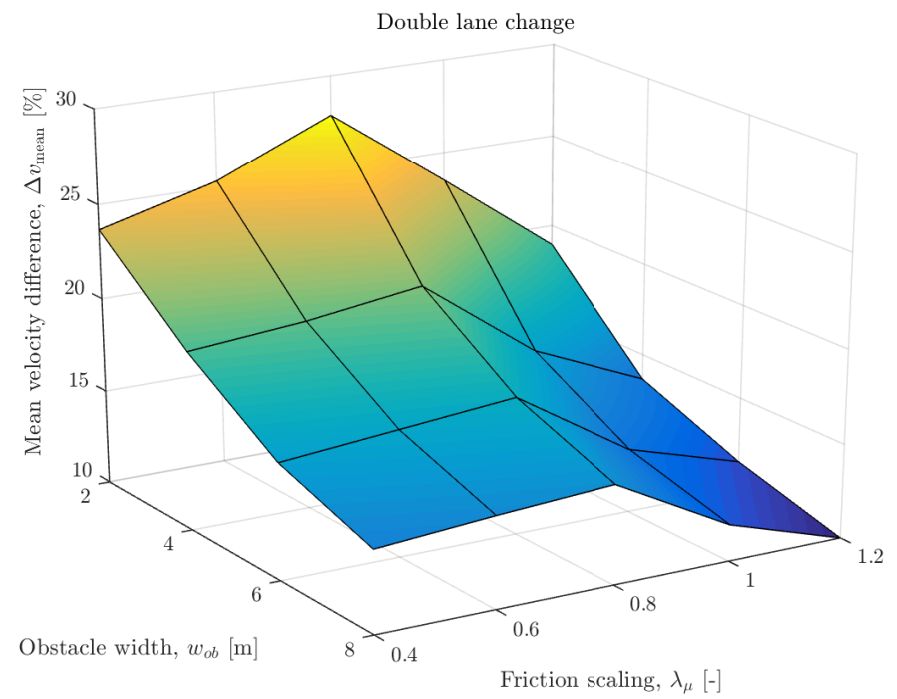

Figure 19. The relative difference of the mean velocity between the double track model and the planar no-slip model in the double lane-change maneuver, for different obstacle widths $w_{o b}$ and friction scaling $\lambda_{\mu}$.

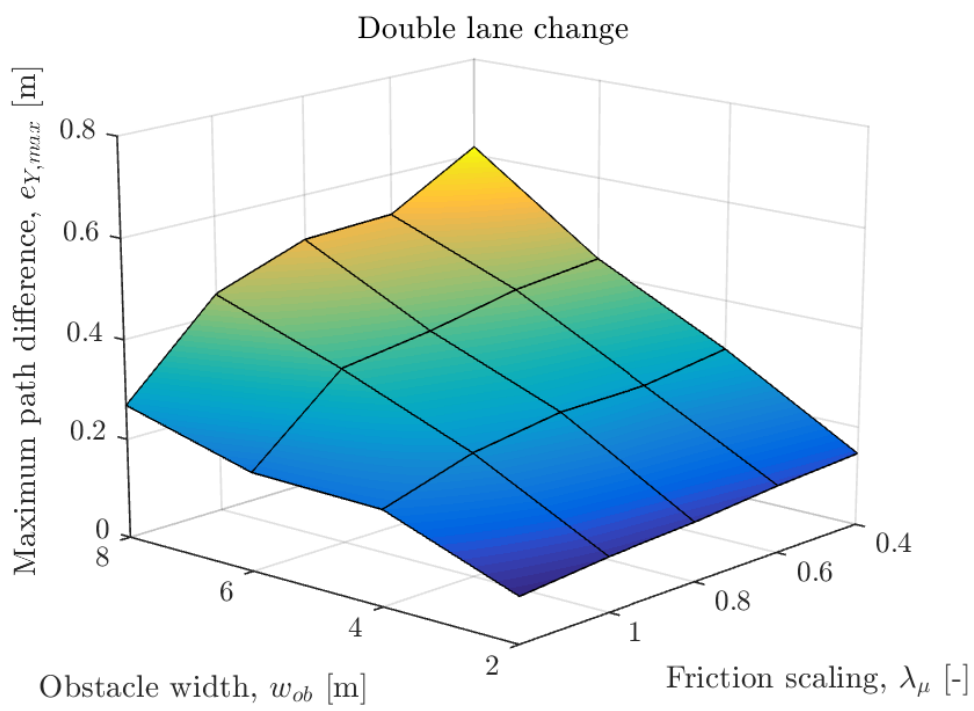

Figure 20. The lateral path difference $e_{Y}$ describes the difference between the paths of the double track model and the planar no-slip model throughout the double lane-change maneuver. Here, the maximum value of $e_{Y}$ is presented for different obstacle widths $w_{o b}$ and friction scaling $\lambda_{\mu}$ in the double lane change.

\author{
西
}


maximum steering rate $\dot{\delta}_{\text {max }}$. Since, $C=\frac{\dot{\psi}}{v}$ and $\dot{\psi}=\frac{v}{l} \delta$, from (7) and (31), the maximum curvature rate for the planar no-slip model is determined by

$$
\left(\frac{\mathrm{d} C}{\mathrm{~d} s}\right)_{\max }^{P N S}=\frac{\dot{\delta}_{\max }}{v l}
$$

which is also what the surface for the planar no-slip model in Figure 21(b) corresponds to. For the double track model, a similar expression for the maximum curvature rate is much more complex to derive due to the amount of variables influencing these characteristics. However, a rough estimate can be computed for a few simple assumptions. The curvature rate can be expressed as

$$
\frac{\mathrm{d} C}{\mathrm{~d} s}=\frac{\ddot{\psi}}{v^{2}},
$$

if the velocity $v$ is assumed constant. The yaw dynamics, derived from (14e), can be simplified and expressed as

$$
I_{z z} \ddot{\psi}=\left(F_{y, 1}+F_{y, 2}\right) l_{f}-\left(F_{y, 3}+F_{y, 4}\right) l_{r} .
$$

The maximum yaw acceleration $\ddot{\psi}_{\max }$ can thus be assumed to be proportional to the friction coefficient,

$$
\ddot{\psi}_{\max } \propto \mu_{y}
$$

from which follows that

$$
\left(\frac{\mathrm{d} C}{\mathrm{~d} s}\right)_{\max }^{D T} \propto \frac{\mu_{y}}{v^{2}}
$$

Since $a_{y}=C v^{2}$ and $\left|a_{y}\right| \leq \mu_{y} g$ when limited by friction, the ratio $\frac{\mu_{y}}{v^{2}}$ will remain constant for a given curvature. This is also seen in Figure $21(\mathrm{~b})$, where $\left(\frac{\mathrm{d} C}{\mathrm{~d} s}\right)_{\max }$ for the double track model is almost completely constant over different friction scaling $\lambda_{\mu}$. Furthermore, the maximum curvature rate increases approximately linearly with the obstacle width $w_{o b}$. This corresponds to the ratio $\frac{1}{v_{\text {mean }}^{2}}$ varying near-to-linearly, where $v_{\text {mean }}$ is the mean velocity of the double track model, listed in Table 7.

The planar no-slip model will always, due to the few limitations it possess, return a driving path with the smallest curvature possible, since this allow the highest velocity. Thus, its path will be consistent over different friction levels, and $C_{\max }$ for the planar no-slip model is therefore constant over $\lambda_{\mu}$ in Figure 21(a). The double track model cannot generate curvature rates of the same magnitude as the planar no-slip model, and therefore has to compensate by later and sharper turning corresponding to larger $C_{\max }$ in Figure 21(a). For both models, $C_{\max }$ is seen to increase for wider obstacles, which is reasonable due to the changed geometry of the maneuver requiring a more extensive avoidance action.

\subsubsection{Double lane change: Some observations and implications}

A valuable observation from the double lane-change results is the relation shown in (50), from which a maximum vehicle velocity can be determined in yaw transients, based on the 


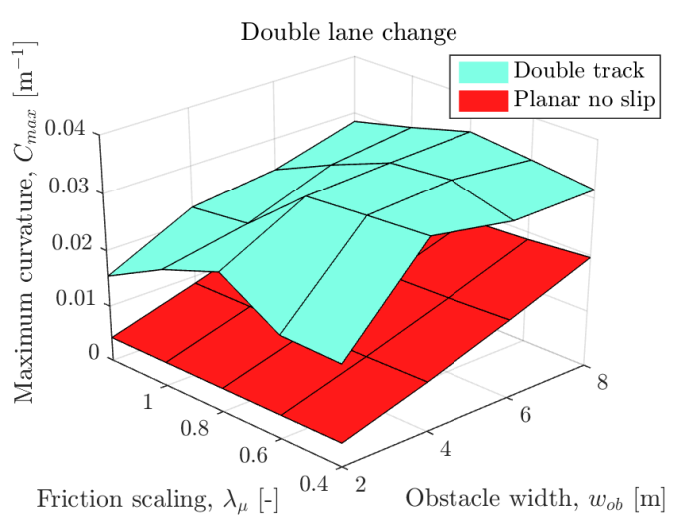

(a) The maximum path curvature $C$ for each model.

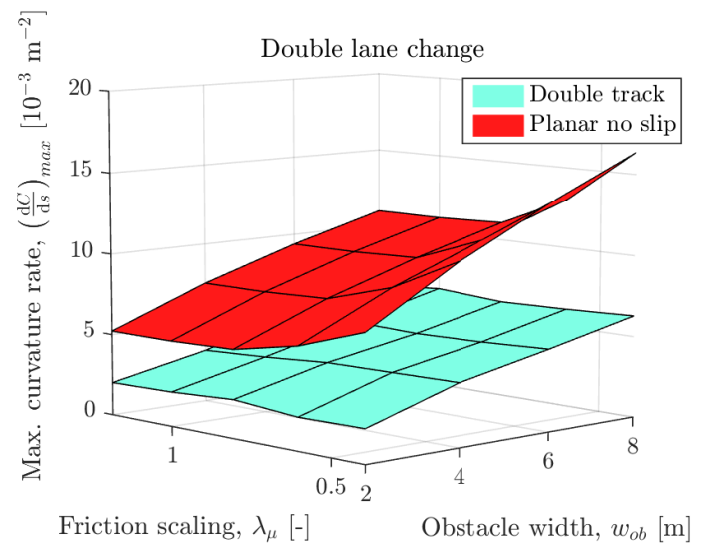

(b) The maximum path-curvature rate $\frac{\mathrm{d} C}{\mathrm{~d} s}$ for each model.

Figure 21. The maximum curvature $C_{\max }$ and curvature rate $\left(\frac{\mathrm{d} C}{\mathrm{~d} s}\right)_{\max }$ of the paths obtained for the double track model and the planar no-slip model, in the double lane-change maneuver, different obstacle widths $w_{o b}$ and friction scaling $\lambda_{\mu}$.

path curvature rate and the road surface friction. Combined with equations representing the stationary cornering capabilities, such as (47) and (48), this can form a basis for path following in avoidance maneuvers.

\section{Discussion}

The results in the previous section can be interpreted in several ways. Here, the double track model is treated as a reference model that is assumed to represent the real world vehicle dynamics accurately. The planar no-slip model and the static model are then evaluated in terms of their ability to represent important characteristics, when exposed to the critical driving scenarios analyzed in this study. It is here important to note that even if the results would indicate, for example, that the static model is capable of predicting the maximum speed profile accurately under certain driving conditions, this does not guarantee the actual vehicle is able to follow that speed, unless it is steered with the optimal control input. However, if the opposite is found, where the double track model is unable to reach the performance of the less complex models, even with optimal steering and braking inputs, then the simplified models can be discarded as model predictors for that particular purpose (if no modifications are made or safety margins added).

On a general note, although the driven path is similar between the models, the control input differs considerably. See the steer angle $\delta$ in Figure 8, 14, and 17. Thus, the simplified models are not suitable for predicting low-level control actions, but works well for overall behavior. Similar attributes were also discovered and discussed in [4].

\section{The significance of path tolerance}

The velocity surface plots in Figures $9(\mathrm{~b})$ and 13 show which of the double track model and the static model is predicting the highest constant velocity in the clothoid turn. Assume the static model is used in an on-board maximum speed-profile estimation algorithm, in a vehicle accurately described by the double track model. Then, if the path properties corresponds to the gray area of either Figure 9(b) or 13 (that is, 
where the static model returns the highest velocity), the vehicle will be unable to negotiate the turn or maneuver at the predicted speed. However, for $R_{\min } \geq 30 \mathrm{~m}$ and $\left(\frac{\mathrm{d} C}{\mathrm{~d} s}\right)_{\text {max }} \geq 0.6 \cdot 10^{-3} \mathrm{~m}^{-2}$, the line dividing the two surfaces is relatively straight. Then, a path tolerance of $e_{\max } \geq 7 \mathrm{~cm}$, under those path characteristics circumstances, will guarantee there exist a control sequence where the vehicle successfully can drive with the predicted velocity.

\section{Brake strategy characteristics}

The braking characteristics in Figures 15 and 16 show distinct variations between the models. Nevertheless, the double track model's surfaces are reasonably flat over large areas, indicating that the optimal strategies can be simple functions of general pathgeometry quantities. More specifically, when to initiate the braking phase is proportional to $R_{\min }$ (see Figure 15) and the time spent during braking proportional to $\sqrt{R_{\min }}$ (see Figure 16). Such relations can be valuable for a velocity or braking controller, where performance similar to the double track model could be obtained with a fraction of the model complexity.

\section{The implication of yaw inertia for different path characteristics}

When subjected to confined maneuvering with quickly altering path curvatures, the yaw inertia has a significant effect on the overall dynamics for the double track model. This can for example be seen in Figure 11, where the double track model spends less time being $L T R$ constrained, since building up yaw rate requires more effort, for the sharper turns with smaller $R_{\min }$. This is somewhat contradicting to [9], where it was concluded that the yaw inertia only has minor effect on the time optimal maneuvering. However, the contrasting outcome is explained by the more demanding maneuver geometries used here.

The maximum curvature rate is, as derived in (50), proportional to the quotient of the available tire-to-ground friction and the squared velocity for the double track model. This expression, combined with (49), can be formalized as a constraint on the steering rate, according to

$$
|\dot{\delta}| \leq \frac{\mu_{y} l}{v} k_{d C}
$$

where the parameter $k_{d C}=2.0$ is estimated from the double lane-change results for the double track model. As an example of the consequence this constraint brings, results obtained for the planar no-slip model with (51) added is shown in Figure 22. Except from this constraint, all model and maneuver properties are identical to the configuration in Figure 17. The maximum lateral path-difference $e_{Y}$ is almost halved, reduced by $11 \mathrm{~cm}$, by introducing the new constraint. To put this into perspective, consider the maximum velocities in Table 6, where increasing the path tolerance from 1 to $10 \mathrm{~cm}$ on average results in a $3.2 \%$ gain in maximum speed. The constraint proposed in (51) is not a universal tool equalizing the planar no-slip model to the considerably more complex double track model. However, it does exemplify that rather simple but clever expressions can be used as constraints for simplified models, to obtain more accurate results without adding much complexity. 

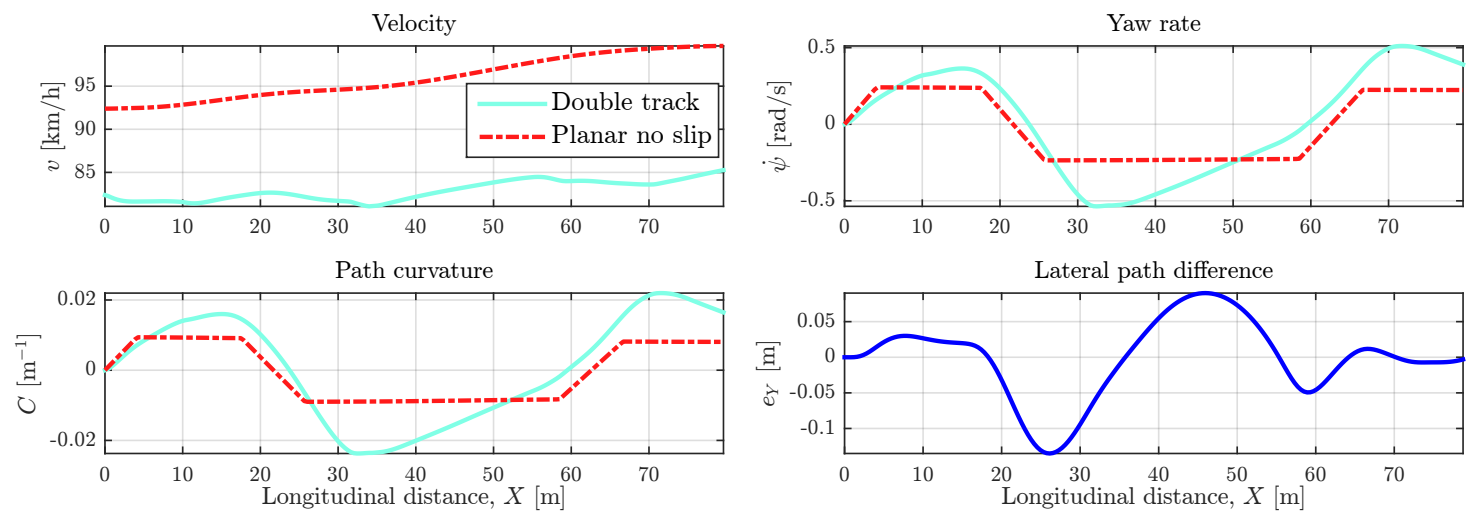

Figure 22. The optimal solutions obtained for the double track model and the planar no-slip model, where (51) has been introduced as a constraint in the planar no-slip model. The maneuver properties are otherwise identical to those in Figure 17, that is, the double lane change with $w_{o b}=4 \mathrm{~m}$ and $\lambda_{\mu}=1.0$.

\section{Conclusions}

Path following is a core component in evasive safety maneuvers and autonomous driving. Motivated by these applications, path following has here been studied considering path tolerance and other path characteristics in relation to main effects in vehicle dynamics. The underlying need for real-time and reasonably high speed calls for quantitative analysis to function as guidance on required model complexity depending on path characteristics. The presentation and discussions in Sections 6 and 7 gave several such results, whereof some deserves to be highlighted since they are valuable in themselves but also illustrates different types of interplay. The finding that a simple model is sufficient if the tolerance is wide enough is important. This was presented in the discussion around Figures $9(\mathrm{~b})$ and 13 . The quantification that only around a decimeter, or less, is enough is striking. In the lower left corner of these figures more tolerance is needed since for higher curvature (lower radius) the yaw inertia of the vehicle comes into play, an interesting illustration of the interplay between path characteristics and vehicle dynamics. The yaw inertia was also important in the results on the connection between rate of change of curvature and available friction in Figure 21 and Equation (50), which also give good guidance.

Overall, in situations where the path planner, due to the type of vehicle operation ranging from good transport roads to more tricky maneuvering, give paths with a wide range of tolerances and characteristics, then the results provided give the basis for path following without going unnecessarily slow.

\section{Funding}

The research presented in this paper has been supported by the iQMatic project funded by the innovation and research program FFI/Vinnova, the Strategic Area for ICT research ELLIIT funded by the Swedish Government, and the Swedish Research Council within the Linnaeus Center CADICS. 


\section{References}

[1] Andersson, J. 2013. A General-Purpose Software Framework for Dynamic Optimization. PhD thesis, Arenberg Doctoral School, KU Leuven, Department of Electrical Engineering (ESAT/SCD) and Optimization in Engineering Center, Kasteelpark Arenberg 10, 3001-Heverlee, Belgium.

[2] Berntorp, K. 2014. Particle Filtering and Optimal Control for Vehicles and Robots. PhD thesis, Department of Automatic Control, Lund University, Sweden.

[3] Berntorp, K., Olofsson, B., Lundahl, K., Bernhardsson, B., and Nielsen, L. 2013. Models and methodology for optimal vehicle maneuvers applied to a hairpin turn. In Proc. Am. Control Conf. (ACC), pages 2139-2146, Washington, DC.

[4] Berntorp, K., Olofsson, B., Lundahl, K., and Nielsen, L. 2014. Models and methodology for optimal trajectory generation in safety-critical roadvehicle manoeuvres. Vehicle System Dynamics, 52(10):1304-1332.

[5] Biegler, L. T. 2010. Nonlinear programming : concepts, algorithms, and applications to chemical processes. MOS-SIAM series on optimization. Philadelphia : Society for Industrial and Applied Mathematics : Mathematical Optimization Society, c2010.

[6] Bobrow, J. E., Dubowsky, S., and Gibson, J. S. 1985. Time-optimal control of robotic manipulators along specified paths. Int. J. Robotics Research, 4(3):3-17.

[7] Buehler, M., Iagnemma, K., and Singh, S. 2007. The 2005 DARPA grand challenge: the great robot race, volume 36. Springer Science \& Business Media.

[8] Buehler, M., Iagnemma, K., and Singh, S. 2009. The DARPA Urban Challenge: Autonomous vehicles in city traffic, volume 56. springer.

[9] Casanova, D., Sharp, R., and Symonds, P. 2000. Minimum time manoeuvring: The significance of yaw inertia. Vehicle System Dynamics, 34(2):77-115.

[10] Cossalter, V., Lio, M. D., Lot, R., and Fabbri, L. 1999. A general method for the evaluation of vehicle manoeuvrability with special emphasis on motorcycles. Vehicle System Dynamics, 31(2):113-135.

[11] Dahlberg, E. 2001. Commercial vehicle stability-focusing on rollover. PhD thesis, KTH.

[12] Gordon, T. and Lidberg, M. 2015. Automated driving and autonomous functions on road vehicles. Vehicle System Dynamics, 53(7):958-994.

[13] Guizzo, E. 2011. How googles self-driving car works. IEEE Spectrum Online, October, 18.

[14] HSL 2013. A collection of Fortran codes for large scale scientific computation, http://www.hsl.rl.ac.uk/.

[15] ISO 3888-2:2011 2011. Passenger cars-Test track for a severe lane-change manoeuvre-Part 2: Obstacle avoidance. International Organization for Standardization, Geneva, Switzerland.

[16] Kapania, N. R. and Gerdes, J. C. 2015. Design of a feedback-feedforward steering controller for accurate path tracking and stability at the limits of handling. Vehicle System Dynamics, 53(12):16871704 .

[17] Lima, P., Trincavelli, M., Martensson, J., and Wahlberg, B. 2015. Clothoid-based model predictive control for autonomous driving. In Control Conference (ECC), 2015 European, pages 2983-2990.

[18] Limebeer, D. and Rao, A. 2015. Faster, higher, and greener: Vehicular optimal control. Control Systems, IEEE, 35(2):36-56.

[19] Lundahl, K., Lee, C. F., Frisk, E., and Nielsen, L. 2015. Analyzing rollover indices for critical truck maneuvers. SAE International Journal of Commercial Vehicles, 8(1):189-196.

[20] Nielsen, L. and Dahl, O. 1990. Torque limited path following by on-line trajectory time scaling. IEEE Transactions on Robotics and Automation, 6(5).

[21] Olofsson, B. 2015. Topics in Machining with Industrial Robot Manipulators and Optimal Motion Control. PhD thesis, Department of Automatic Control, Lund University, Sweden.

[22] Pacejka, H. B. 2006. Tyre and Vehicle Dynamics. Butterworth-Heinemann, Oxford, United Kingdom, second edition.

[23] Preston-Thomas, J. and Woodrooffe, J. 1990. A feasibility study of a rollover warning device for heavy trucks. Transport Canada Publication No. TP 10610E.

[24] Scattolini, R. 2009. Architectures for distributed and hierarchical model predictive control a review. Journal of Process Control, 19(5):723 - 731.

[25] Sharp, R. S. and Peng, H. 2011. Vehicle dynamics applications of optimal control theory. Vehicle System Dynamics, 49(7):1073-1111.

[26] Wächter, A. and Biegler, L. T. 2006. On the implementation of an interior-point filter line-search algorithm for large-scale nonlinear programming. Mathematical Programming, 106(1):25-57. 